bioRxiv preprint doi: https://doi.org/10.1101/2020.06.29.178129; this version posted June 30, 2020. The copyright holder for this preprint

(which was not certified by peer review) is the author/funder, who has granted bioRxiv a license to display the preprint in perpetuity. It is made available under aCC-BY-NC-ND 4.0 International license.

\title{
Heh2/Man1 may be an evolutionarily conserved sensor of NPC assembly state
}

2

3 Sapan Borah\%, David J. Thaller\%, Zhanna Hakhverdyan ${ }^{\star}$, Elisa C. Rodriguez $\%$, Michael P.

4 Rout ${ }^{\star}$, Megan C. King\% and C. Patrick Lusk\%*

5

$6 \%$ Department of Cell Biology, Yale School of Medicine, 295 Congress Avenue, New Haven, CT,

$7 \quad 06520$

8 \&The Rockefeller University, 1230 York Avenue, New York, NY, 10065

9

10 *Correspondence to C. Patrick Lusk

11 patrick.lusk@yale.edu

12

13

14 


\section{Abstract}

Integral membrane proteins of the Lap2-emerin-MAN1 (LEM) family have emerged as important

17 components of the inner nuclear membrane (INM) required for the functional and physical

18 integrity of the nuclear envelope. However, like many INM proteins, there is limited understanding of the biochemical interaction networks that enable LEM protein function. Here, we show that Heh2/Man1 can be affinity purified with major scaffold components of the nuclear pore complex (NPC), specifically the inner ring complex, in evolutionarily distant yeasts.

22 Interactions between Heh2 and nucleoporins is mediated by its C-terminal winged-helix (WH)

23 domain and are distinct from interactions required for INM targeting. Disrupting interactions

24 between Heh2 and the NPC leads to NPC clustering. Interestingly, Heh2's association with

25 NPCs can also be broken by knocking out Nup133, a component of the outer ring that does not physically interact with Heh2. Thus, Heh2's association with NPCs depends on the structural

27 integrity of both major NPC scaffold complexes. We propose a model in which Heh2 acts as a sensor of NPC assembly state, which may be important for NPC quality control mechanisms and the segregation of NPCs during cell division. 


\section{Introduction}

34 The eukaryotic genome is enclosed by a nuclear envelope that is contiguous with the endoplasmic reticulum (ER). Despite this continuity, the nuclear envelope contains a unique proteome that defines its function as a selective barrier. This barrier not only establishes nuclear-cytoplasmic compartmentalization but also directly impacts genome organization and function at the nuclear periphery (Mekhail and Moazed, 2010; Taddei and Gasser, 2012; Buchwalter et al., 2019). The key elements of this biochemical specialization are the nuclear pore complexes (NPCs), which control nucleocytoplasmic molecular exchange, and proteins specifically associated with the inner and outer nuclear membranes (INM and ONM)(Ungricht and Kutay, 2017; Hampoelz et al., 2019). While ONM proteins generally act as adaptors that connect the cytoskeleton to the nucleus (Burke and Roux, 2009), INM protein function is less well defined. This is due in part to challenges inherent with defining biochemical interactions between low abundance integral membrane proteins that exist within a complex and integrated network of peripheral chromatin and nuclear scaffold proteins like the lamins (outside of yeasts). Nonetheless, there is confidence that there are several dozen integral INM proteins with the most evolutionarily conserved families being the LAP2-emerin-MAN1 (LEM) proteins and the SUN family proteins (Mans et al., 2004; Ungricht and Kutay, 2015).

LEM family proteins are so named for their LEM domain, a short $\sim 40$ amino acid helixextension-helix motif that, at least in higher eukaryotes, binds to barrier to autointegration factor (BAF)(Furukawa, 1999; Cai et al., 2007). As there is no BAF in yeasts, the LEM domain must possess other conserved functions, which may more directly relate to genome integrity, ensuring the stability of repetitive DNA (Mekhail et al., 2008), and also contributing to the mechanical integrity of the nucleus (Schreiner et al., 2015). There are up to seven LEM domain proteins in humans but in the two most commonly used yeast models, Saccharomyces cerevisiae (Sc) and Schizosaccharomyces pombe (Sp) there are only two: ScHeh1(Src1)/SpHeh1(Lem2) and ScHeh2/SpHeh2(Man1)(Barton et al., 2015). Of these two, ScHeh1 and SpHeh1 are likely orthologs derived from a common ancestor, while ScHeh2 and SpHeh2 resulted from independent duplication events of their respective paralogs ScHeh1 and SpHeh1 (Rhind et al., 2011; Gonzalez et al., 2012). Despite their independent evolutionary history, there is evidence that Heh2 in both yeasts specifically makes functional connections with NPCs. For example, in S. cerevisiae, we demonstrated synthetic genetic interactions between genes encoding NPC components (nucleoporins or nups), and HEH2 (Yewdell et al., 2011). In the S. pombe cousin, S. japonicus, it has also been suggested that Heh2 supports 
connections between chromatin and NPCs to support their segregation between daughter cells in mitosis (Yam et al., 2013). However, the underlying biochemical connections between Heh2 and the NPC are not understood.

Understanding the nature of the connections between Heh2 and the NPC may also help illuminate mechanisms underlying the biogenesis of NPCs. As the total proteome, interactome and structure of NPCs have come to light, it is now understood that the enormous (50-100 MD) NPC is built from a relatively small ( 30) number of nups (Hampoelz et al., 2019). These nups are organized into modular subcomplexes that, in multiples of 8 , assemble the 8-fold radially symmetric NPC scaffold composed of inner and outer ring complexes (IRC and ORC), the central transport channel and asymmetric (perpendicular to the plane of the nuclear envelope) cytosolic filaments/mRNA export platform and nuclear basket (Kosinski et al., 2016; Kim et al., 2018). How NPCs are assembled in space and time during interphase remains ill-defined, but likely begins within the nucleus at the INM (Marelli et al., 2001; Makio et al., 2009; Yewdell et al., 2011; Mészáros et al., 2015; Otsuka et al., 2016). The recruitment of nups to an assembly site occurs alongside membrane-remodeling that evaginates the INM and ultimately drives fusion with the ONM (Otsuka et al., 2016). Consistent with an inside-out model, the cytosolicfacing mRNA export platform is likely added at a terminal step in NPC assembly (Otsuka et al., 2016; Onischenko et al., 2017). In genetic backgrounds where the cytoplasmic-facing mRNA export platform is not assembled, herniations or blebs are observed over assembling NPCs, which may reflect defects in INM-ONM fusion and/or the triggering of NPC assembly quality control pathways (Thaller and Lusk, 2018).

Both Heh1 and Heh2 have been implicated in mechanisms of NPC assembly quality control in which they regulate the recruitment of the endosomal sorting complexes required for transport (ESCRT) to the nuclear envelope (Webster et al., 2014, 2016; Thaller et al., 2019). One early model suggested that Heh2 may differentially bind to NPC assembly intermediates over fully formed NPCs (Webster et al., 2014). However, this has yet to be formally interrogated. In order to be more incisive as to how Heh2 impacts NPC function, here we have thoroughly analyzed the biochemical interaction network of endogenous Heh2. Using two evolutionary distant yeasts, we show that Heh2 can co-purify with the NPC's IRC. These interactions do not require the LEM domain or any INM targeting sequences but instead depend on a C-terminal domain predicted to fold into a winged helix (WH)(Caputo et al., 2006). Further, by decoupling NPC clustering from perturbations to NPC structure, we demonstrate that Heh2 associates with NPCs in vivo. 
knocking out Nup133, a nucleoporin of the ORC, suggesting that Heh2's association with the NPC depends on its structural integrity. Taken together, we suggest a model in which Heh2 may be a sensor of NPC assembly state.

\section{Results}

\section{Heh2 binds to specific nups in evolutionarily distant yeasts}

To better define the interacting partners of Heh1 and Heh2, we performed one-step affinity purifications of Heh1-TAP and Heh2-TAP (produced at endogenous levels) from cryolysates derived from logarithmically growing budding yeast (Hakhverdyan et al., 2015). As shown in Fig. 1A, we did not detect any obvious stoichiometric binding partners of Heh1-TAP despite robust recovery of the fusion protein. In marked contrast, Heh2-TAP co-purified with at least 8 additional proteins, which were visible by SDS-PAGE and Coommassie blue staining of bound fractions. Excision of these bands followed by mass spectrometric (MS) protein identification revealed that Heh2 binds to the IRC of the NPC and a subset of cytosolic-facing nups, including Nup159, Nup188, Nup192, Nup170, Pom152, Nup157, Nup116, Nic96 and Nsp1. For context, we have colored the identified nups in a diagram of a single spoke from the budding yeast NPC structure (Kim et al., 2018) in Fig. 1A.

We were next curious whether Heh2's association with the NPC was also observed in other yeast species where the NPC structure is different than in budding yeast. For example, fission yeast NPCs are made up of a similar catalogue of nups (Baï et al., 2004; Chen et al., 2004; Asakawa et al., 2014), but there is evidence that there is asymmetry with respect to the ORC, which contains 16 copies (instead of 8 ) of the " $Y$ " complex on the nucleoplasmic side of the NPC (Asakawa et al., 2019). Of additional interest, although HEH1 in both S. cerevisiae and S. pombe is derived from a common ancestor, these yeasts are separated by $\sim 500$ million years of evolution (Rhind et al., 2011). Intriguingly, and in contrast, ScHEH2 and SpHEH2 arose from distinct duplication events (Mans et al., 2004), and might therefore be expected to carry out distinct functions.

Interestingly however, despite this unique evolutionary history, the affinity-purifications of SpHeh2-TAP and SpHeh1-TAP were qualitatively similar to the $S$. cerevisiae versions with SpHeh1-TAP co-purifying with few specific proteins (compare to the WT control) and SpHeh2TAP with several specific species (Fig. 1B). Note that SpHeh2-TAP is proteolytically sensitive 
and is purified both as a full length ( $115 \mathrm{kDa})$ and a smaller ( 65 kDa) form (Fig. 1B). Nonetheless, like its distant S. cerevisiae cousin, the SpHeh2-complex consisted of essentially the same subset of inner ring nups including Nup184, Nup186, Nup155, Pom152, Npp106, Nup98 and Nup97 (Fig. 1B). To facilitate a comparison, the S. cerevisiae homologues are listed next to the identified S. pombe nups in Fig. 1B. Thus, despite the distinct duplication events that gave rise to $H E H 2$ in both species, the physical association of Heh2 with the IRC likely points to an important and conserved function that was likely shared by a common ancestor before being independently specialized in the two species lineages.

\section{Heh2 fails to interact with NPCs lacking Nup133}

That Heh2 binds to nups suggests that it may be a component of the NPC. To assess this possibility, we next examined the distribution of Heh2-GFP at the nuclear envelope alongside an NPC marker, Nup82-mCherry. We also took advantage of a standard approach of knocking out NUP133, which leads to NPC clustering and facilitates co-localization analysis, as individual NPCs cannot be resolved with conventional light microscopy (Doye et al., 1994; Pemberton et al., 1995; Li et al., 1995; Aitchison et al., 1995; Heath et al., 1995). Consistent with prior work (Yewdell et al., 2011), we observed a punctate NPC-like distribution of Heh2-GFP at the nuclear envelope of otherwise WT cells, which exhibited some co-localization with Nup82-mCherry (Fig. 2A). Indeed, when we quantified the correlation between the GFP and mCherry fluorescence at each pixel along the nuclear envelope of 20 cells, we observed a modest positive correlation ( $r$ $=0.39$; Fig. 2B). In marked contrast, deletion of NUP133 led to a striking anti-correlation between Nup82-mCherry and Heh2-GFP ( $r=-0.27)$, which was obvious in the micrographs where Heh2-GFP was diminished or undetectable at the Nup82-mCherry clusters (Fig. 2A, B, bottom panels). We note further that Heh2-GFP is no longer punctate along the nuclear envelope in nup $133 \Delta$ cells, which suggests that there may in fact be an association with NPCs (as supported by the biochemistry) but that this interaction is broken without Nup133.

To continue with the exploration of potential functional commonalities between ScHeh2 and SpHeh2, we also tested whether deletion of the orthologous S. pombe Nup132 impacted SpHeh2-GFP distribution (Baï et al., 2004). As has been reported by others, SpHeh2 also has a punctate distribution evocative of NPCs (Fig. 2C)(Gonzalez et al., 2012; Steglich et al., 2012). Consistent with this, we observed coincidence between SpHeh2-GFP and SpNup107-mCherry fluorescence with a correlation value of $r=0.49$ (Fig. 2D, top). Interestingly, as in S. cerevisiae, deletion of Nup132 lead to a clear anti-correlation $(r=-0.03)$ of the SpHeh2-GFP and 
SpNup107-mCherry signals, suggesting that their physical interaction could be disrupted (Fig. 2D, bottom). Remarkably, this anti-correlation was observed even with minimal clustering of SpNup107-mCherry in this strain (Fig. 2C). Thus, this result reinforces that disrupting NPC structure by deleting a critical ORC component compromises Heh2's ability to interact with NPCs in both organisms.

\section{Heh2 co-localizes with NPCs}

To reconcile the apparent inconsistency between the affinity purifications, which suggested that Heh2 binds NPCs, and the lack of Heh2-GFP co-clustering with nups in nup133 $\triangle$ strains, we sought an orthogonal approach to assess Heh2-GFP co-localization with NPCs that were not missing key structural components. In prior work, we observed that the anchor-away approach (Haruki et al., 2008)(Fig. 3A) can drive rapid NPC clustering through the rapamycin-induced dimerization of a Nsp1-FRB fusion that was incorporated into NPCs (and likely exposed to the cytosol) with Pma1-FKBP12 (a plasma membrane anchor, Fig. 3A) within 15 min (Colombi et al., 2013). The rapidity of this response strongly suggested that fully formed NPCs are driven into clusters independent of NPC mis-assembly. Further, we did not detect any removal of Nsp1-FRB from NPCs under these conditions (Colombi et al., 2013). Consistent with this, we assessed the co-localization of Nup82-GFP with Nup170-mCherry in strains expressing Nsp1FRB and Pma1-FKPB12 in the presence of carrier alone (DMSO) or rapamycin. As expected, both of the fluorescent proteins localized in a punctate distribution at the nuclear envelope in the presence of DMSO with a significant $r=0.48$ positive correlation between the GFP and mCherry fluorescence (Fig. 3B, far right panel). Upon addition of rapamycin, we observed rapid clustering and concurrent co-localization of both signals along the nuclear envelope, which was evident in the coincidence of the GFP and mCherry fluorescence peaks of line profiles along the nuclear envelope and a correlation that increased to $r=0.74$ (Fig. 3B, middle and right panels).

We next tested how this approach to NPC clustering influenced Heh2-GFP localization. As a control, we also assessed the distribution of Heh1-GFP, which does not stably interact with nups (Fig. 1A). As shown in Fig. 3C, the addition of rapamycin lead to the clear co-localization of Heh2-GFP and Nup170-mCherry. This again was evident through the examination of line profiles of a representative nuclear envelope where there was coincidence between the peaks of the GFP and mCherry fluorescence and further supported by the increased positive correlation of GFP and mCherry fluorescence (from $r=0.18$ to $r=0.64$; Fig. 3C, middle and 
right panels). Note, however, that unlike the comparison between the two nups (Fig. 3B), there are peaks of Heh2-GFP fluorescence that are not coincident with the NPC clusters (Fig. 3C, arrowheads in line profiles). Thus, while it is clear that Heh2-GFP associates with NPCs, there is also an additional pool of Heh2-GFP at the INM. Last, we did not observe similar effects with Heh1-GFP, which failed to cluster with NPCs (Fig. 3D) or correlate with their distribution ( $r=-$ 0.01)(Fig. 3D, right panel). Thus, this NPC clustering approach more faithfully mirrored our biochemical analysis of both Heh1 and Heh2 and supports the interpretation that Heh2 is a shared component of NPCs and the INM.

\section{Inhibition of NPC assembly reduces the Heh2 pool bound to NPCs}

A model in which there are two pools of Heh2 was further supported by experiments where we reduced NPC number by inhibiting NPC assembly. For example, by again leveraging the anchor-away strategy, we inhibited NPC assembly by trapping newly synthesized Nup192-FRBGFP for $3 \mathrm{~h}$ (Colombi et al., 2013). Under these conditions, there is a reduction of NPCs that is reflected by lower levels of Nup192-FRB-GFP at the nuclear envelope and a concomitant accumulation of newly synthesized Nup192-FRB-GFP at the plasma membrane (Fig. 4A, B, rapamycin panels). In this scenario, we tested whether Nup192-FRB-GFP and Heh2-mCherry co-localized at the nuclear envelope (Fig. 4B). As a control, we also tested co-localization with Pom152-mCherry (Fig. 4A). While Pom152-mCherry distribution was similar to Nup192-FRBGFP with line profiles showing coincidence between mCherry and GFP fluorescence peaks along the nuclear envelope (Fig. 4A, far right), there were clear gaps in the Nup192-FRB-GFP signal that were filled by Heh2-mCherry (Fig. 4B, see arrowheads). This result is also represented in line profiles across the nuclear envelope where the Heh2-mCherry signal fills areas that are devoid of GFP-peaks (Fig. 4B, right bottom panel). Importantly, however, a subset of Nup192-FRB-GFP peaks that likely correspond to NPCs that were assembled prior to rapamycin addition still coincided with Heh2-mCherry peaks (Fig. 4B, right bottom panel). Thus, these data are consistent with the interpretation that inhibition of NPC assembly leads to a decrease in the pool of Heh2 bound to NPCs (due to their reduced number) and an increase in the free pool at the INM. This conclusion is further supported by affinity-purifications of Heh2TAP from Nup192-FRB-GFP strains under the same conditions. While in DMSO-treated conditions the expected IRC profile of nups was detected (Fig. 4C), upon inhibition of NPC assembly with rapamycin, we observed a $\sim 2-3$ fold reduction of these nups (orange line in densitometry plot at right) while the total amount of Heh2-TAP affinity purified remained 
unchanged (Fig. 4C). Thus, we favor a model in which Heh2 remains capable of binding to the IRC in fully formed NPCs, even when their number is decreased upon assembly inhibition.

\section{Heh2's association with NPCs depends on the integrity of the NPC scaffold}

If Heh2 binds the IRC, it remained unclear why deletion of NUP133 abrogated Heh2's NPC association, as the IRC is expected to be intact in this background. Thus, to rule out that Heh2 may be binding IRC nups outside of the context of fully formed NPCs, we directly tested whether deletion of NUP133 lead to a loss of Heh2 IRC binding. Strikingly, affinity purifications of Heh2-TAP in nup133 $\Delta$ cells did not reveal any obvious binding partners, with the potential exception of Nup159, further supporting the in vivo evidence that the structurally deficient nup133 $\triangle$ NPCs are incompetent for binding Heh2 (Fig. 5A). This result is illustrated as a loss of the colored Heh2-interacting nups within the context of a side and center view of a NPC spoke in Fig. 5B. Consistent with the conserved lack of colocalization of scHeh2-GFP and spHeh2GFP with NPCs in the absence of Nup133/Nup132, we also observed a loss of nups in affinitypurified fractions of SpHeh2-TAP from nup 132 $\Delta$ extracts (Fig. 5C).

We next explored the hierarchy of physical interactions that control Heh2's association with the IRC by affinity-purifying Heh2-TAP from several IRC nup deletion backgrounds. Interestingly, and in contrast with the deletion of NUP133, we were unable to define any single knockout of an inner ring nup that fully broke Heh2's biochemical association with this complex. For example, in cases where we deleted the genes encoding Nup157 or Pom152, we observed the discrete loss of these, and only these, proteins from bound fractions (Fig. 5A, B). Deletion of NUP170 and NUP188 led to a more severe disruption of nups bound to Heh2, but in these cases, Pom152 and $a$ band at the molecular weight of Nup159 remained (Fig. 5A, B). Thus, it seems likely that Heh2 makes several direct connections to nups in the IRC, with the most obvious candidates being Pom152, Nup170 and/or Nup188. Heh2 may also directly bind to Nup159, although this association alone is insufficient to maintain association with the NPC in vivo (Fig. 2A).

Our inability to fully break interactions between Heh2 and the NPC by abrogating single nups within the IRC was further supported by the lack of any major changes to Heh2-GFP distribution

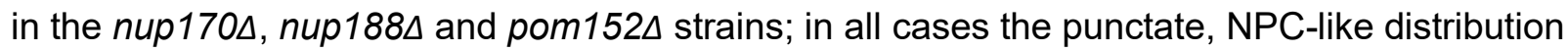
of Heh2-GFP was retained (Fig. 5D). The one potential exception here was that, in addition to the punctate nuclear envelope distribution, a cortical ER pool of Heh2-GFP could be discerned specifically in nup $170 \Delta$ strains (Fig. 5D, arrowhead). These data are consistent with prior work 
demonstrating that Nup170 is uniquely required for the efficient targeting of overexpressed Heh2 to the INM (King et al., 2006). Thus, we suggest that, with the exception of Nup170, the physical interactions with the IRC described here are dispensable for INM targeting. Such an assertion is further supported by the exclusive nuclear envelope localization of Heh2-GFP in nup133 cells where virtually all of its biochemical interactions to the NPC are broken (Fig. 2A). These data thus make the prediction that the INM targeting and NPC-binding elements of Heh2 are distinct.

\section{The conserved WH domain of Heh2 is required for NPC association}

To explore the possibility that INM targeting and NPC-binding may require unique structural elements of Heh2, we generated truncations of Heh2 where the N-terminal nucleoplasmic domain (which contains the INM-targeting information (King et al., 2006; Meinema et al., 2011)) and the C-terminal WH domains are deleted (Fig. 6A). Interestingly, deletion of the N-terminus did not impact binding to nups, as a similar (if more robust) profile of the IRC was recovered in affinity purifications of heh2-(316-663)-TAP (Fig. 6B). These data suggest that Heh2 can reach the NPC (or at least bind to nups) in the absence of its N-terminal INM targeting domain. In marked contrast, deletion of the WH domain, which does not impact INM targeting (Meinema et al., 2011), led to a striking reduction of nup binding (Fig. 6B). These results were also mirrored in vivo. For example, compared with Heh2-GFP, heh2-(1-570)-GFP did not exhibit a punctate distribution at the nuclear envelope (Fig. 6C), which was quantified as a reduced coefficient of variation of the fluorescence signal along the nuclear envelope (Fig. 6D). Consistent with the idea that this change in localization of heh2-(1-570)-GFP was due to a loss of its interaction with NPCs, it also failed to cluster with NPCs in the Nsp1-FRB NPC clustering assay (Fig. 6E) with no positive correlation between heh2-(1-570)-GFP and Nup170-mCherry signals in either DMSO $(r=0.0)$ or rapamycin $(r=-0.08)$ treated cells (Fig. 6F). Thus, the WH domain of Heh2 is the major determinant of its association with NPCs.

\section{WH-domain-mediated interactions with NPCs are required for normal NPC distribution}

As the Heh2 WH-domain was specifically required for Heh2-binding to NPCs, but not for INM targeting, there was an opportunity to define a putative NPC-specific function for Heh2. Indeed, deletion of $H E H 2$ leads to a marked clustering of Nup82-GFP, which was quantified as a 
291

292

293

294

295

296

297

298

299

300

301

302

303

304

305

306

307

308

309

310

311

312

313

314

315

316

317

318

319

320

321

322

coefficient of variation (CV) of the fluorescence along the nuclear envelope that was approximately double the value in WT cells (Fig. 6G, H). To directly test whether this phenotype was due to a loss of nup-binding, we assessed the distribution of Nup82-GFP in cells expressing heh2-(1-570). Indeed, as shown in Fig. 6G, this targeted abrogation of the nupbinding WH domain also resulted in a clear redistribution of Nup82-GFP, showing a clustering coefficient nearly identical to that seen in heh2 $\Delta$ cells $($ Fig. $6 \mathrm{H})$. Thus, interactions between Heh2 and the NPC are required for normal NPC distribution.

Interestingly, expression of heh2-(316-663) from its endogenous locus also impacted NPC distribution, but with a unique phenotype. Because this truncation of Heh2 lacks its INM targeting information, this fusion will be mislocalized to the endoplasmic reticulum (King et al., 2006; Meinema et al., 2011). In these cells, Nup84-GFP accumulated in clusters at the nuclear envelope but also appeared within cytosolic foci (Fig. 6I, arrowheads) in $\sim 17 \%$ of cells. Together then, these data support a model in which both the N-terminal and C-terminal domains of Heh2 are important for NPC distribution, however, the underlying mechanisms behind these alterations are unique and reflect either too little (in the case of heh2-(1-570)) and likely inappropriate (in the case of heh2-(316-663) interactions with nups.

\section{Discussion}

We have explored the physical and functional relationship between the integral INM protein Heh2 and the NPC. This study was motivated by our prior discovery of predominantly genetic interactions between HEH2 and nup genes (Yewdell et al., 2011), in addition to other work considering Heh2 as a factor in a NPC assembly surveillance pathway (Webster et al., 2014, 2016). In the latter, we imparted Heh2 the ability to discern between NPC assembly intermediates and fully formed NPCs. This concept was centered, in part, on data showing that Heh2 does not associate with clustered NPCs in nup133 $\Delta$ strains, which was interpreted in a model where Heh2 does not bind to fully formed NPCs. We now provide a more nuanced explanation for these data, as deletion of Nup133 breaks Heh2's otherwise robust physical association with the NPC (Fig. 5A). Thus, in light of the new data presented here, a reconsideration of the role of Heh2 in NPC biology is needed. Given these new observations, we suggest that Heh2 likely binds to fully formed NPCs. Several data support this assertion including: 1) The biochemical interactions that suggest the formation of a stable complex between Heh2 and the IRC (Fig. 1A, B). 2) The maintenance of these interactions even upon 
NPC assembly inhibition (Fig. 4C) and 3) The punctate distribution of Heh2 at steady-state and upon clustering of functional NPCs driven by the anchoring of Nsp1-FRB (Fig. 3C).

325 Despite the demonstration that Heh2 associates with NPCs, several new conundrums arise as a 326 consequence of this work. The first is that we do not observe any robust physical association 327 between Heh2 and the ORC, and yet, deletion of Nup133 leads to a loss of Heh2 binding to the 328 NPC (Fig. 5A). In contrast, we cannot break Heh2's association with NPCs by knocking out any individual component of the IRC (Fig. 5A, D). While the latter can be explained in a model where Heh2 makes several direct but redundant connections with nups, likely Pom152 and Nup170 and/or Nup188, the former is more challenging to interpret. Several potential models can be considered. The first deals with the very nature of nup133 $\mathrm{NPC}$ clustering, which has so far remained only partially explained on a mechanistic level. For example, one thought is that the association of NPCs with the pore membrane is destabilized without the amphipathic helix/ALPS motif in Nup133 (Drin et al., 2007), which may lead to pore clustering (FernandezMartinez et al., 2012). In such a scenario, given that it is an integral membrane protein, Heh2's interactions with the NPC may depend on the presence of specific lipids or membrane curvature (or both) at the pore membrane. Alternatively, the clustering itself may sterically preclude an interaction with Heh2. It is also possible that the IRC may not be fully functional or be structurally perturbed in this context. Regardless of the underlying mechanism, as Heh2's association with the NPC ultimately depends on the function of both of its major scaffold complexes (i.e. the IRC and ORC), we favor a model in which Heh2 can, through a mechanism that remains to be defined, "sense" the structural integrity of the NPC.

344 A model in which Heh2 is a sensor for the NPC scaffold fits within a quality control mechanism

345 framework. For example, recent work suggests that NPC clustering can facilitate clearance of 346 NPCs by autophagy (Lee et al., 2020). Thus, it is tempting to speculate that damage to the NPC scaffold may trigger the release of Heh2, which would in turn lead to the clustering of damaged NPCs. Such an idea is supported by the clustering that we observe in contexts where Heh2NPC interactions are abrogated (Fig. 6G, H). Similarly, as we have previously reported, NPC clustering may also be an input that ensures that damaged or malformed NPCs are not transmitted to daughter cells (Webster et al., 2014). Thus, the consistent theme is that breaking 352 interactions between Heh2 and NPCs may be an input to their segregation and/or clearance. A corollary to this is that Heh2 bound to NPCs may in fact promote the inheritance of functional

354 NPCs. This may be best illustrated by work from S. japonicus where it was demonstrated that 355 the Heh2 orthologue contributes to anchoring NPCs to chromatin to promote their proper 
segregation between daughters (Yam et al., 2013). Indeed, our observation that Heh2 also engaged in interactions with the IRC in $S$. pombe argues that it supports a fundamental role(s) across diverse yeasts.

How, then, do interactions between Heh2 and NPCs ensure proper NPC distribution? We speculate that in the absence of mechanisms to keep NPCs apart, NPCs have an inherent conformation or affinity that drives their clustering. In this scenario, binding NPCs to INM proteins could help ensure their physical segregation. Although this could be envisaged purely as a steric inhibition of NPC-NPC interactions, we favor the concept that the distribution of NPCs and other elements of the nuclear architecture are co-dependent. Indeed, our prior work suggests that SpHeh2 antagonizes the flow of chromatin into nuclear deformations (Schreiner et al., 2015), in essence maintaining normal chromatin distribution at the nuclear periphery, a direct corollary of the effect here on NPC distribution. As SpHeh2 binds both chromatin (Gonzalez et al., 2012; Steglich et al., 2012) and NPCs (this work), it is tempting to speculate that it supports the normal organization of NPCs and chromatin by dynamically linking these two major structural components of the nucleus. This concept is consistent with evidence in mammalian cells where NPCs are well established to be anchored to the lamin network (Daigle et al., 2001; Maeshima et al., 2006; Xie and Burke, 2017; Kittisopikul et al., 2020). In scenarios in which this lamin connection is broken, for example in lamin knockouts, NPCs also cluster together (Xie and Burke, 2017; Kittisopikul et al., 2020). Although NPCs are more dynamic along the nuclear envelope in budding yeast (Belgareh and Doye, 1997; Bucci and Wente, 1997), their interactions with chromatin through multiple mechanisms (Luthra et al., 2007; TanWong et al., 2009) could nonetheless contribute to their normal distribution. Whether clustering has an impact on NPC function per se remains ill defined, although one could speculate that NPC clustering has a more profound impact on the NPC's roles in chromatin organization and gene expression as opposed to nuclear transport (Capelson et al., 2010; Raices and D’Angelo, 2017).

One particularly interesting feature of our analysis of Heh2 is that the NPC binding and INM targeting sequences are distinct and on two physically separated domains. Certainly there is evidence from both genetic and biochemical analyses where the function of specific domains of the LEM domain proteins can be separated (Grund et al., 2008; Yewdell et al., 2011; Barrales et al., 2016; Hirano et al., 2018; Thaller et al., 2019; von Appen et al., 2020). However, we wonder whether there are functional implications for the integration of these two interaction platforms, which could place Heh2 in a tug-of-war between its residence bound to the NPC and its release 
bioRxiv preprint doi: https://doi.org/10.1101/2020.06.29.178129; this version posted June 30, 2020. The copyright holder for this preprint (which was not certified by peer review) is the author/funder, who has granted bioRxiv a license to display the preprint in perpetuity. It is made available under aCC-BY-NC-ND 4.0 International license.

389 to the INM. This would be yet another example in an emerging theme for these LEM domain

390 proteins in which they bridge distinct sets of physical interactions to maintain the dynamic

391 organization of the nuclear envelope system. 


\section{Figure legends}

393

394

395

396

397

398

399

400

401

402

403

404

405

406

407

408

409

410

411

412

413

414

415

416

417

418

419

420

Figure1. Heh2 binds to specific nups in evolutionary distant yeasts

(A) Heh2 specifically binds the IRC. Affinity purifications were performed from cell extracts derived from strains expressing endogenous Heh1-TAP or Heh2-TAP or from WT cells (no TAP). Bound proteins were separated by SDS-PAGE and visualized by Coomassie staining. Numbers at left indicate position of MW standards in KD. Heh1-TAP and Heh2-TAP are indicated, and colored circles demark proteins identified by MS from Heh2-TAP lane, as indicated in key. This color scheme is also used to indicate positions of nups within a single spoke of the NPC structure (from PDBDEV_00000010; Kim et al., 2018). ORC is outer ring complex, IRC is inner ring complex.

(B) As in A but affinity purifications performed from S. pombe cell extracts. The corresponding S. cerevisiae homologues of the identified S. pombe nups are also listed.

\section{Figure 2. Heh2 fails to interact with NPCs lacking Nup133}

(A) Deconvolved fluorescence micrographs of Heh2-GFP and Nup82-mCherry with merge in WT and nup133 $\Delta$ strains. Arrowheads point to regions depleted of Heh2-GFP that contain Nup82-mCherry in a cluster. Scale bar is $5 \mu \mathrm{m}$.

(B) Scatterplot with Pearson correlation coefficient ( $r$ ) of Heh2-GFP and Nup82-mCherry fluorescence intensity (in arbitrary units, a.u.) along the nuclear rim of 20 cells, from two independent experiments.

(C) Deconvolved fluorescence micrographs of SpHeh2-GFP, and SpNup107-mCherry with merge in WT and nup132 $\Delta$ S. pombe cells. Scale bar is $5 \mu \mathrm{m}$.

(D) Scatterplot with Pearson correlation coefficient ( $r$ ) of SpHeh2-GFP and SpNup107-mCherry fluorescence intensity (in arbitrary units, a.u.) along the nuclear rim of 20 cells, from two independent experiments.

\section{Figure 3. Heh2 associates with NPCs in vivo.}

(A) Schematic of NPC clustering assay mediated by the rapamycin-induced dimerization of Nsp1-FRB (at the NPC) and Pma1-FKBP12. $\mathrm{N}$ is nucleus, $\mathrm{V}$ is vacuole. 
421 (B-D) Left: Deconvolved fluorescence micrographs of indicated GFP tagged proteins and

422 Nup170-mCherry as a NPC marker with merge in cells treated with DMSO (carrier) or

423 rapamycin for $15 \mathrm{~min}$. Scale bar is $5 \mu \mathrm{m}$. Middle: Line profiles of fluorescence intensity of GFP

424 and mCherry fusions (in arbitrary units, a.u.) along the nuclear envelope of a single cell. Right:

425 Scatterplot with Pearson correlation coefficient $(r)$ of GFP and mCherry fluorescence intensity

426 (in arbitrary units, a.u.) along the nuclear rim of 30 cells, from three independent experiments.

Figure 4. Inhibition of NPC assembly reduces the Heh2-nup bound pool

429

430

431

432

433

434

435

436

437

438

439

440

441

442

443

444

445

446

447

448

449

450

(A, B) Deconvolved fluorescence micrographs of Nup192-FRB-GFP with either Pom152mCherry or Heh2-mCherry with merge after treating cells with DMSO (carrier) or rapamycin for $3 \mathrm{~h}$ to inhibit NPC assembly. Note accumulation of newly synthesized Nup192-FRB-GFP at the plasma membrane as it binds to the Pma1-FKBP12 anchor. Arrowheads point to Heh2-mCherry at the nuclear envelope that is resolvable from Nup192-FRB-GFP signal. Scale bar is $2 \mu \mathrm{m}$. At right are line profiles of GFP and mCherry fluorescence intensity (in arbitrary units, a.u.) along the nuclear envelope of single cells corresponding to DMSO (top) and rapamycin (bottom) conditions.

(C) Inhibiting NPC assembly reduces Heh2-IRC binding. Affinity purifications were performed from cell extracts derived from cells expressing Heh2-TAP with Nup192-FRB-GFP and Pma1FKBP12 treated with carrier (DMSO) alone, or with rapamycin (rap) to inhibit NPC assembly. Bound proteins were separated by SDS-PAGE and visualized with Coomassie. Position of MW markers $(\mathrm{kD})$ are indicated at left and proteins are marked with colored circles that denote their identity as per key at right. Densitometry of the protein staining of the DMSO (black) and rapamycin (orange) lanes on right.

\section{Figure 5. NPC scaffold integrity affects Heh2's association with NPCs}

(A) Affinity purifications were performed from cell extracts derived from the indicated nup gene deletion strains expressing endogenous Heh2-TAP or from WT cells (no TAP). Bound proteins were separated by SDS-PAGE and visualized by Coomassie staining. Numbers at left indicate position of MW standards in kD. Proteins are marked with colored circles that denote their identify as per key at right. 
451 (B) The nups affinity purified from the indicated genetic backgrounds in A are placed within a

452 single spoke of the NPC structure (from PDBDEV_00000010; Kim et al., 2018) in side and

453 center views. Individual nups are colored as in the key in A.

454 (C) As in A but affinity purifications performed from S. pombe cell extracts.

455 (D) Deconvolved fluorescence micrographs of Heh2-GFP in indicated strain backgrounds. White 456 arrowhead points to Heh2-GFP fluorescence at the cortical ER in nup170 $\Delta$ cells. Scale bars are $4575 \mu \mathrm{m}$.

458

Figure 6. The WH domain of Heh2 is required for its association with NPCs

(A) Schematic of Heh2 and Heh2 truncations showing the LEM (Lap2-Emerin-Man1) domain, a amino acid numbers. INM, inner nuclear membrane.

(B) Affinity purifications were performed from cell extracts derived from strains expressing the indicated TAP fusions or from WT cells (no TAP). Bound proteins were separated by SDSPAGE and visualized by Coomassie staining. Numbers at left indicate position of MW standards 467 in kD. Red circles denote position of TAP-fusions.

(C) Deconvolved fluorescence micrographs of Heh2-GFP or heh2-(1-570)-GFP and the NPC marker Nup82-mCherry, with merge. Scale bar is $5 \mu \mathrm{m}$.

(D) To quantitatively evaluate the distribution of Heh2-GFP and heh2-(1-570)-GFP, a coefficient of variation (CV) of the GFP fluorescence along the nuclear envelope was calculated. Individual

472 CV values (multiplied by 100) were plotted with mean and SD from 60 cells, from three

473 independent experiments. $p$ values were calculated from Student's t-test where ${ }^{* * *}$ indicates $p$ $474 \leq 0.0001$.

475 (E) Deconvolved fluorescence micrographs of heh2-(1-570)-GFP and Nup170-mCherry with 476 merge in cells expressing Nsp1-FRB and Pma1-FKBP12. Cells were treated with carrier 477 (DMSO) or rapamycin. Addition of rapamycin leads to NPC clustering as described in Fig. 3A. 478 Scale bar is $5 \mu \mathrm{m}$.

479 (F) Scatterplot with Pearson correlation coefficient $(r)$ of heh2-(1-570)-GFP and Nup170480 mCherry fluorescence intensity (in arbitrary units, a.u.) along the nuclear envelope of 30 cells 
481 from three independent experiments like that shown in E. Values are from cells from DMSO

482 (top) and rapamycin-treated (bottom) conditions.

483 (G) The WH domain of Heh2 is required for normal NPC distribution. Deconvolved fluorescence 484 micrographs of Nup82-GFP in indicated strain backgrounds. Scale bar is $5 \mu \mathrm{m}$.

485 (H) To quantitatively evaluate the distribution of Nup82-GFP in the indicated strains, a 486 coefficient of variation (CV) of the GFP fluorescence along the nuclear envelope was calculated. 487 Individual CV values (multiplied by 100) were plotted with mean and SD from 60 cells, from 488 three independent experiments. $p$ values were calculated from one-way ANOVA with Tukey's 489 post-hoc test where ns is $p>0.05,{ }^{* * * *} p \leq 0.0001$.

490 (I) Deconvolved fluorescence micrographs of Nup84-GFP in WT and cells where HEH2 is 491 replaced by heh2-(316-663). Arrowheads point to cytosolic Nup84-GFP foci. Scale bar is $5 \mu \mathrm{m}$.

492 (J) Quantification of the percentage of cells where Nup84-GFP is found in the cytosol from 493 experiment in I. Error bars are SD from four independent experiments. $p$ values were calculated 494 with unpaired t-test where ${ }^{* *}$ indicates $p \leq 0.01$.

495

496

497

498

499

500

501

502

503

504

505

506

507 


\section{Materials and methods}

509 Yeast culture and strain generation

510 All yeast strains used in this study are listed in Table S1. S. cerevisiae strains were grown in

511 YPD consisting of $1 \%$ Yeast extract (BD), 2\% Bacto-peptone (BD) and, 2\% D-glucose (Sigma).

512 For microscopy experiments, YPD was supplemented with $0.025 \%$ adenine hemi-sulfate

513 (Sigma). Yeast cells were grown at $30^{\circ} \mathrm{C}$ to mid-log phase, unless otherwise stated.

514 Transformation of S. cerevisiae cells, mating, sporulation and tetrad-dissections were carried

515 out using standard protocols (Amberg et al., 2005). Deletion and truncation of yeast ORFs and

516 tagging of ORFs with fluorescent protein genes, FRB and TAP-tags was performed utilizing the

517 pFA6a or pK3F plasmid templates (Longtine et al., 1998; Zhang et al., 2017).

518 S. pombe strains were grown in YE5S media consisting of 5\% Yeast extract (BD), 30\% D-

519 glucose (Sigma) and 1.25\% SP complete supplements (adenine hemisulfate, L-histidine

520 hydrochloride monohydrate, L-leucine, L-lysine hydrochloride and uracil) from Sunrise Science

521 products, at $30^{\circ} \mathrm{C}$. S. pombe strains were crossed and maintained utilizing standard media and

522 techniques as described in (Moreno et al., 1991). PCR based gene disruption and tagging were

523 performed utilizing pFA6a plasmid templates (Bähler et al., 1998; Hentges et al., 2005).

525 Plasmids

526 All plasmids used in this study are listed in Table S2. The pFA6a-TAP-his3MX6 and pFA6a-

527 TAP-TRP1 plasmids were constructed as follows: the TAP coding sequence was PCR-amplified

528 from chromosomal DNA from a strain expressing Heh2-TAP (SBCPL42, Dharmacon yeast

529 resources) using Phusion High fidelity DNA polymerase (New England BioLabs) and cloned into 530 the $P$ acl and Ascl sites of pFA6a-his3MX6 and pFA6a-TRP1.

531 pFA6a-3xHA-FRB-GFP-his3MX6 was generated by Gibson Assembly (New England BioLabs).

532 The 3xHA epitope coding sequence was PCR-amplified from pFA6a-3xHA-hisMX6 (Longtine et 533 al., 1998) using Q5 DNA polymerase (New England BioLabs) and assembled into pFA6a-FRB534 GFP-hisMX6, or pFA6a-FRB-hisMX6 (EUROSCARF) digested with Sall and Pacl. 
To affinity purify TAP-fusions, S. cerevisiae strains were grown overnight and $2 \mathrm{ml}$ of culture was diluted into $1 \mathrm{I}$ of YPD the next morning and grown for 20-24 $\mathrm{h}$ to late log phase $\left(\mathrm{OD}_{600} \sim 2\right)$. S. pombe cells were grown overnight and transferred to fresh medium the next morning to an $\mathrm{OD}_{600}$ of 0.1 and grown for $7 \mathrm{~h}$. S. pombe cells were further diluted to an $\mathrm{OD}_{600}$ of 0.01 in $1 \mathrm{I}$ YES medium and grown for another 18-20 h. Both S. cerevisiae and S. pombe cells were grown at $30^{\circ} \mathrm{C}$ at $200 \mathrm{rpm}$ and cells were harvested by centrifugation. Cells were washed with ice-cold water once, collected by centrifugation and resuspended in $100 \mu \mathrm{l}$ freezing solution (20 mM HEPES, pH 7.4, 1.2\% polyvinylpyrrolidone and protease inhibitor cocktail [Sigma]) per g of cells. The cell slurry was snap-frozen in liquid nitrogen immediately. The frozen cell pellets were cryomilled 6 times at $30 \mathrm{~Hz}$ for 3 min in a Retsch MM400 mixer mill and stored at $-80^{\circ} \mathrm{C}$.

To perform immunoaffinity purifications, $200 \mathrm{mg}$ of frozen yeast grindate was resuspended in 4times volume of homogenization buffer (400 mM Na 3 Cit, pH 8.0, 10 mM Deoxy Big CHAP) and protease inhibitor cocktail at room temperature. The homogenate was clarified by centrifugation at $16,000 \mathrm{~g}$ for $10 \mathrm{~min}$ at $4^{\circ} \mathrm{C}$. The soluble fraction was incubated with $25 \mu \mathrm{l}$ of Rabbit-lgG coated Dynabeads for $1 \mathrm{~h}$ at $4^{\circ} \mathrm{C}$ under gentle rotation. After binding, beads were collected on a magnetic rack and washed three times with $500 \mu$ ice-cold homogenization buffer. The proteins were eluted by incubating beads with $20 \mu \mathrm{l}$ of 1 X NuPAGE lithium dodecyl sulfate sample buffer (Invitrogen) at room temperature for $10 \mathrm{~min}$. The eluate was separated on a magnetic rack and further incubated with $50 \mathrm{mM} \mathrm{DTT}$ at $70^{\circ} \mathrm{C}$ for $10 \mathrm{~min}$. The eluted proteins were separated on a 4-12\% NuPAGE gel (Novex) and stained with Imperial protein stain (Thermo Scientific). The proteins of interest were excised for identification by MS.

\section{Conjugation of Dynabeads with Rabbit IgG}

Purified rabbit IgG (Sigma, 15006) was dissolved in $0.1 \mathrm{M}$ sodium phosphate buffer, $\mathrm{pH} 7.4$, to a final concentration of $1 \mathrm{mg} / \mathrm{ml}$. The lgG solution was filtered through a $0.22 \mu \mathrm{m}$ syringe filter and mixed with an equal volume of $3 \mathrm{M}\left(\mathrm{NH}_{4}\right)_{2} \mathrm{SO}_{4}$. For conjugation, $100 \mathrm{mg}$ of Dynabeads $\circledR \mathrm{M}-270$ Epoxy (Invitrogen) were transferred to a $15 \mathrm{ml}$ centrifuge tube, suspended in $6 \mathrm{ml} 0.1 \mathrm{M}$ sodium phosphate buffer, $\mathrm{pH} 7.4$ and incubated at room temperature for $15 \mathrm{~min}$ on a tube rotator. The beads were collected on a magnetic rack, the buffer aspirated and beads were washed again with $0.1 \mathrm{M}$ sodium phosphate buffer, $\mathrm{pH} 7.4$ by vortexing. The buffer was removed and beads were resuspended in $2 \mathrm{ml}$ of $\mathrm{lgG}$ solution and incubated at $30^{\circ} \mathrm{C}$ for $65-70 \mathrm{~h}$ on a tube rotator. The beads were separated on a magnetic rack and quickly washed with $100 \mathrm{mM}$ glycine, $\mathrm{pH}$ 2.5 , followed by a wash with $10 \mathrm{mM}$ Tric- $\mathrm{HCl}, \mathrm{pH}$ 8.8. Beads were again washed quickly with freshly prepared $100 \mathrm{mM}$ Triethylamine and followed by 4 washes with PBS for 5 min each and 
570 one wash with PBS with $0.5 \%$ Triton X-100 for 15 min. The beads were washed one final time

571 with PBS, collected on a magnetic rack and resuspended in $667 \mu$ PBS with $50 \%$ glycerol.

\section{Anchor-away experiments}

573 The anchor-away experiments were performed as described by Haruki et al., 2008. Briefly,

574 strains expressing Nup-FRB fusions and Pma1-FKPB12 in HHY110 (tor1-1 fpr1 $\Delta$ ) were

575 incubated with a final concentration of $1 \mu \mathrm{g} / \mathrm{ml}$ rapamycin for $30 \mathrm{~min}$ (to cluster NPCs in the

576 context of Nsp1-FRB) or $3 \mathrm{~h}$ to inhibit assembly (Nup192-FRB).

577 Fluorescence microscopy, image processing and analysis

578 Fluorescence micrographs were acquired on a DeltaVision microsope (Applied Precision, GE

579 Healthcare) with a 100x, 1.4 NA objective (Olympus). The images were captured with a

580 CoolSnapHQ2 CCD camera (Photometrics). Fluorescence micrographs were deconvolved with

581 the iterative algorithm sofWoRx. 6.5.1 (Applied Precision, GE Healthcare).

582 Clustering of NPCs was quantified as described previously (FernandezMartinez et al., 2012): A

583 6-pixel wide freehand line was drawn along the nuclear envelope contour and mean

584 fluorescence intensities were measured using FIJI/ImageJ (Schindelin et al., 2012). Clustering

585 was assessed by calculating the coefficient of variance (SD/mean X 100) of the fluorescence

586 intensities at the nuclear envelope.

587 Modeling of NPC spokes

588 Color coding of an isosurface representation of individual nup densities as assigned in Kim et al.

5892018 within an individual spoke of the NPC from the PDB DEV ID:00000010 was completed

590 using ChimeraX (UCSF) (Goddard et al., 2018). 
bioRxiv preprint doi: https://doi.org/10.1101/2020.06.29.178129; this version posted June 30,2020. The copyright holder for this preprint

(which was not certified by peer review) is the author/funder, who has granted bioRxiv a license to display the preprint in perpetuity. It is made available under aCC-BY-NC-ND 4.0 International license.

\section{Acknowledgements}

593 We thank Jean Kanyo and the Yale Keck Biotechnology Resource Laboratory for help with MS

594 analysis. We would like to thank for Valérie Doye for S. pombe strains. We would like to thank

595 the members of the Lusk and King laboratories for critical input on experimental design and data

596 analysis. This work was supported by the NIH: R01 GM105672 to CPL, R21 HG006742 to CPL

597 and MCK, and R01 GM112108 and P41 GM109824 to MPR and the NSF: EFMA-1806504 to

598 MCK. 


\section{References}

Aitchison, J.D., G. Blobel, and M.P. Rout. 1995. Nup120p: a yeast nucleoporin required for NPC distribution and mRNA transport. J. Cell Biol. 131:1659-1675. doi:10.1083/jcb.131.6.1659.

Amberg, D.C., D.J. Burke, and J.N.J.N. Strathern. 2005. Methods in Yeast Genetics: A Cold Spring Harbor Laboratory Course Manual, 2005 Edition. 2005 ed. Cold Spring Harbor Laboratory Press.

von Appen, A., D. LaJoie, I.E. Johnson, M.J. Trnka, S.M. Pick, A.L. Burlingame, K.S. Ullman, and A. Frost. 2020. LEM2 phase separation promotes ESCRT-mediated nuclear envelope reformation. Nature. 582. doi:10.1038/s41586-020-2232-x.

Asakawa, H., T. Kojidani, H.J. Yang, C. Ohtsuki, H. Osakada, A. Matsuda, M. Iwamoto, Y. Chikashige, K. Nagao, C. Obuse, Y. Hiraoka, and T. Haraguchi. 2019. Asymmetrical localization of nup107-160 subcomplex components within the nuclear pore complex in fission yeast. PLoS Genet. 15:1-30. doi:10.1371/journal.pgen.1008061.

Asakawa, H., H.J. Yang, T.G. Yamamoto, C. Ohtsuki, Y. Chikashige, K. Sakata-Sogawa, M. Tokunaga, M. Iwamoto, Y. Hiraoka, and T. Haraguchi. 2014. Characterization of nuclear pore complex components in fission yeast Schizosaccharomyces pombe. Nucl. (United States). 5. doi:10.4161/nucl.28487.

Bähler, J., J.Q. Wu, M.S. Longtine, N.G. Shah, A. McKenzie, A.B. Steever, A. Wach, P. Philippsen, and J.R. Pringle. 1998. Heterologous modules for efficient and versatile PCRbased gene targeting in Schizosaccharomyces pombe. Yeast. 14:943-951. doi:10.1002/(SICI)1097-0061(199807)14:10<943::AID-YEA292>3.0.CO;2-Y.

Baï, S.W., J. Rouquette, M. Umeda, W. Faigle, D. Loew, S. Sazer, and V. Doye. 2004. The Fission Yeast Nup107-120 Complex Functionally Interacts with the Small GTPase Ran/Spi 1 and Is Required for mRNA Export, Nuclear Pore Distribution, and Proper Cell Division. Mol. Cell. Biol. 24:6379-6392. doi:10.1128/mcb.24.14.6379-6392.2004.

Barrales, R.R., M. Forn, P.R. Georgescu, Z. Sarkadi, and S. Braun. 2016. Control of heterochromatin localization and silencing by the nuclear membrane protein Lem2. Genes Dev. 30:133-148. doi:10.1101/gad.271288.115.

Barton, L.J., A.A. Soshnev, and P.K. Geyer. 2015. Networking in the nucleus: a spotlight on LEM-domain proteins. Curr. Opin. Cell Biol. 34:1-8. 
doi:https://doi.org/10.1016/j.ceb.2015.03.005.

630

631

632

633

634

635

636

637

638

639

640

641

642

643

644

645

646

647

648

649

650

651

652

653

654

655

656

657

658

Belgareh, N., and V. Doye. 1997. Dynamics of nuclear pore distribution in nucleoporin mutant yeast cells. J. Cell Biol. 136:747-759. doi:10.1083/jcb.136.4.747.

Bucci, M., and S.R. Wente. 1997. In vivo dynamics of nuclear pore complexes in yeast. J. Cell Biol. 136:1185-99.

Buchwalter, A., J.M. Kaneshiro, and M.W. Hetzer. 2019. Coaching from the sidelines: the nuclear periphery in genome regulation. Nat. Rev. Genet. 20:39-50. doi:10.1038/s41576018-0063-5.

Burke, B., and K.J. Roux. 2009. Nuclei Take a Position: Managing Nuclear Location. Dev. Cell. 17:587-597. doi:10.1016/j.devcel.2009.10.018.

Cai, M., Y. Huang, J.-Y. Suh, J.M. Louis, R. Ghirlando, R. Craigie, and G.M. Clore. 2007. Solution NMR structure of the barrier-to-autointegration factor-Emerin complex. J. Biol. Chem. 282:14525-14535. doi:10.1074/jbc.M700576200.

Capelson, M., C. Doucet, and M.W. Hetzer. 2010. Nuclear pore complexes: guardians of the nuclear genome. Cold Spring Harb. Symp. Quant. Biol. 75:585-597. doi:10.1101/sqb.2010.75.059.

Caputo, S., J. Couprie, I. Duband-Goulet, E. Kondé, F. Lin, S. Braud, M. Gondry, B. Gilquin, H.J. Worman, and S. Zinn-Justin. 2006. The carboxyl-terminal nucleoplasmic region of MAN1 exhibits a DNA binding winged helix domain. J. Biol. Chem. 281:18208-18215. doi:10.1074/jbc.M601980200.

Chen, X.Q., X. Du, J. Liu, M.K. Balasubramanian, and D. Balasundaram. 2004. Identification of genes encoding putative nucleoporins and transport factors in the fission yeast Schizosaccharomyces pombe: a deletion analysis. Yeast. 21:495-509. doi:10.1002/yea.1115.

Colombi, P., B.M. Webster, F. Fröhlich, and C. Patrick Lusk. 2013. The transmission of nuclear pore complexes to daughter cells requires a cytoplasmic pool of Nsp1. J. Cell Biol. 203:215-232. doi:10.1083/jcb.201305115.

Daigle, N., J. Beaudouin, L. Hartnell, G. Imreh, E. Hallberg, J. Lippincott-Schwartz, and J. Ellenberg. 2001. Nuclear pore complexes form immobile networks and have a very low turnover in live mammalian cells. J. Cell Biol. 154:71-84. doi:10.1083/jcb.200101089. 
Doye, V., R. Wepf, and E.C. Hurt. 1994. A novel nuclear pore protein Nup133p with distinct roles in poly $(\mathrm{A})+\mathrm{RNA}$ transport and nuclear pore distribution. EMBO J. 13:6062-6075.

Drin, G., J.F. Casella, R. Gautier, T. Boehmer, T.U. Schwartz, and B. Antonny. 2007. A general amphipathic $\alpha$-helical motif for sensing membrane curvature. Nat. Struct. Mol. Biol. 14:138-146. doi:10.1038/nsmb1194.

Fernandez-Martinez, J., J. Phillips, M.D. Sekedat, R. Diaz-Avalos, J. Velazquez-Muriel, J.D. Franke, R. Williams, D.L. Stokes, B.T. Chait, A. Sali, and M.P. Rout. 2012. Structurefunction mapping of a heptameric module in the nuclear pore complex. J. Cell Biol. 196:419-434. doi:10.1083/jcb.201109008.

Furukawa, K. 1999. LAP2 binding protein 1 (L2BP1/BAF) is a candidate mediator of LAP2chromatin interaction. J. Cell Sci. 112:2485-2492.

Goddard, T.D., C.C. Huang, E.C. Meng, E.F. Pettersen, G.S. Couch, J.H. Morris, and T.E. Ferrin. 2018. UCSF ChimeraX: Meeting modern challenges in visualization and analysis. Protein Sci. 27:14-25. doi:10.1002/pro.3235.

Gonzalez, Y., A. Saito, and S. Sazer. 2012. Fission yeast Lem2 and Man1 perform fundamental functions of the animal cell nuclear lamina. Nucleus. 3:60-76. doi:10.4161/nucl.18824.

Grund, S.E., T. Fischer, G.G. Cabal, O. Antúnez, J.E. Pérez-Ortín, and E. Hurt. 2008. The inner nuclear membrane protein Src1 associates with subtelomeric genes and alters their regulated gene expression. J. Cell Biol. 182:897-910. doi:10.1083/jcb.200803098.

Hakhverdyan, Z., M. Domanski, L.E. Hough, A.R.A.A. Oroskar, A.R.A.A. Oroskar, S. Keegan, D.J. Dilworth, K.R. Molloy, V. Sherman, J.D. Aitchison, D. Fenyö, B.T. Chait, T.H. Jensen, M.P. Rout, and J. Lacava. 2015. Rapid, optimized interactomic screening. Nat. Methods. 12:553-560. doi:10.1038/nmeth.3395.

Hampoelz, B., A. Andres-Pons, P. Kastritis, and M. Beck. 2019. Structure and Assembly of the Nuclear Pore Complex. Annu. Rev. Biophys. 48:515-536. doi:10.1146/annurev-biophys052118-115308.

Haruki, H., J. Nishikawa, and U.K. Laemmli. 2008. The Anchor-Away Technique: Rapid, Conditional Establishment of Yeast Mutant Phenotypes. Mol. Cell. 31:925-932. doi:10.1016/j.molcel.2008.07.020.

Heath, C. V, C.S. Copeland, D.C. Amberg, V. Del Priore, M. Snyder, and C.N. Cole. 1995. 
Nuclear pore complex clustering and nuclear accumulation of poly $(\mathrm{A})+\mathrm{RNA}$ associated with mutation of the Saccharomyces cerevisiae RAT2/NUP120 gene. J. Cell Biol. 131:1677-1697. doi:10.1083/jcb.131.6.1677.

Hentges, P., B. Van Driessche, L. Tafforeau, J. Vandenhaute, and A.M. Carr. 2005. Three novel antibiotic marker cassettes for gene disruption and marker switching in Schizosaccharomyces pombe. Yeast. 22:1013-1019. doi:10.1002/yea.1291.

Hirano, Y., Y. Kinugasa, H. Asakawa, Y. Chikashige, C. Obuse, T. Haraguchi, and Y. Hiraoka. 2018. Lem2 is retained at the nuclear envelope through its interaction with Bqt4 in fission yeast. Genes to Cells. 23:122-135. doi:10.1111/gtc.12557.

Kim, S.J., J. Fernandez-Martinez, I. Nudelman, Y. Shi, W. Zhang, B. Raveh, T. Herricks, B.D. Slaughter, J.A. Hogan, P. Upla, I.E. Chemmama, R. Pellarin, I. Echeverria, M. Shivaraju, A.S. Chaudhury, J. Wang, R. Williams, J.R. Unruh, C.H. Greenberg, E.Y. Jacobs, Z. Yu, M.J. De La Cruz, R. Mironska, D.L. Stokes, J.D. Aitchison, M.F. Jarrold, J.L. Gerton, S.J. Ludtke, C.W. Akey, B.T. Chait, A. Sali, and M.P. Rout. 2018. Integrative structure and functional anatomy of a nuclear pore complex. Nature. 555:475-482. doi:10.1038/nature26003.

King, M.C., C.P. Lusk, and G. Blobel. 2006. Karyopherin-mediated import of integral inner nuclear membrane proteins. Nature. 442:1003-1007. doi:10.1038/nature05075.

Kittisopikul, M., T. Shimi, M. Tatli, J.R. Tran, Y. Zheng, O. Medalia, K. Jaqaman, S.A. Adam, and R.D. Goldman. 2020. Nuclear Lamins A/C and B1 Provide a Structural Framework That Organizes and Anchors Nuclear Pore Complexes. bioRxiv. 2020.04.03.022798. doi:10.1101/2020.04.03.022798.

Kosinski, J., S. Mosalaganti, A. von Appen, R. Teimer, A.L. DiGuilio, W. Wan, K.H. Bui, W.J.H. Hagen, J.A.G. Briggs, J.S. Glavy, E. Hurt, and M. Beck. 2016. Molecular architecture of the inner ring scaffold of the human nuclear pore complex. Science. 352:363-365. doi:10.1126/science.aaf0643.

Lee, C.W., F. Wilfling, P. Ronchi, M. Allegretti, S. Mosalaganti, S. Jentsch, M. Beck, and B. Pfander. 2020. Selective autophagy degrades nuclear pore complexes. Nat. Cell Biol. 22:159-166. doi:10.1038/s41556-019-0459-2.

Li, O., C. V Heath, D.C. Amberg, T.C. Dockendorff, C.S. Copeland, M. Snyder, and C.N. Cole. 1995. Mutation or deletion of the Saccharomyces cerevisiae RAT3/NUP133 gene causes 
temperature-dependent nuclear accumulation of poly(A)+ RNA and constitutive clustering of nuclear pore complexes. Mol. Biol. Cell. 6:401-417. doi:10.1091/mbc.6.4.401.

Longtine, M.S., A. McKenzie, D.J. Demarini, N.G. Shah, A. Wach, A. Brachat, P. Philippsen, and J.R. Pringle. 1998. Additional modules for versatile and economical PCR-based gene deletion and modification in Saccharomyces cerevisiae. Yeast. 14:953-961. doi:10.1002/(SICI)1097-0061(199807)14:10<953::AID-YEA293>3.0.CO;2-U.

Luthra, R., S.C. Kerr, M.T. Harreman, L.H. Apponi, M.B. Fasken, S. Ramineni, S. Chaurasia, S.R. Valentini, and A.H. Corbett. 2007. Actively transcribed GAL genes can be physically linked to the nuclear pore by the SAGA chromatin modifying complex. J. Biol. Chem. 282:3042-3049. doi:10.1074/jbc.M608741200.

Maeshima, K., K. Yahata, Y. Sasaki, R. Nakatomi, T. Tachibana, T. Hashikawa, F. Imamoto, and N. Imamoto. 2006. Cell-cycle-dependent dynamics of nuclear pores: Pore-free islands and lamins. J. Cell Sci. 119:4442-4451. doi:10.1242/jcs.03207.

Makio, T., L.H. Stanton, C.C. Lin, D.S. Goldfarb, K. Weis, and R.W. Wozniak. 2009. The nucleoporins Nup170p and Nup157p are essential for nuclear pore complex assembly. J. Cell Biol. 185:459-473. doi:10.1083/jcb.200810029.

Mans, B.J., V. Anantharaman, L. Aravind, and E. V. Koonin. 2004. Comparative genomics, evolution and origins of the nuclear envelope and nuclear pore complex. Cell Cycle. 3:1625-1650. doi:10.4161/cc.3.12.1316.

Marelli, M., C.P. Lusk, H. Chan, J.D. Aitchison, and R.W. Wozniak. 2001. A link between the synthesis of nucleoporins and the biogenesis of the nuclear envelope. J. Cell Biol. 153:709-724. doi:10.1083/jcb.153.4.709.

Meinema, A.C., J.K. Laba, R.A. Hapsari, R. Otten, F.A.A. Mulder, A. Kralt, G. Van Den Bogaart, C.P. Lusk, B. Poolman, and L.M. Veenhoff. 2011. Long unfolded linkers facilitate membrane protein import through the nuclear pore complex. Science (80-. ). 333:90-93. doi:10.1126/science.1205741.

Mekhail, K., and D. Moazed. 2010. The nuclear envelope in genome organization, expression and stability. Nat. Rev. Mol. Cell Biol. 11:317-328. doi:10.1038/nrm2894.

Mekhail, K., J. Seebacher, S.P. Gygi, and D. Moazed. 2008. Role for perinuclear chromosome tethering in maintenance of genome stability. Nature. 456:667-670. 
doi:10.1038/nature07460.

Mészáros, N., J. Cibulka, M.J. Mendiburo, A. Romanauska, M. Schneider, and A. Köhler. 2015. Nuclear pore basket proteins are tethered to the nuclear envelope and can regulate membrane curvature. Dev. Cell. 33:285-298. doi:10.1016/j.devcel.2015.02.017.

Moreno, S., A. Klar, and P. Nurse. 1991. Molecular genetic analysis of fission yeast Schizosaccharomyces pombe. Methods Enzymol. 194:795-823. doi:10.1016/00766879(91)94059-I.

Onischenko, E., J.H. Tang, K.R. Andersen, K.E. Knockenhauer, P. Vallotton, C.P. Derrer, A. Kralt, C.F. Mugler, L.Y. Chan, T.U. Schwartz, and K. Weis. 2017. Natively Unfolded FG Repeats Stabilize the Structure of the Nuclear Pore Complex. Cell. 171:904-917.e19. doi:10.1016/j.cell.2017.09.033.

Otsuka, S., K.H. Bui, M. Schorb, M. Julius Hossain, A.Z. Politi, B. Koch, M. Eltsov, M. Beck, and J. Ellenberg. 2016. Nuclear pore assembly proceeds by an inside-out extrusion of the nuclear envelope. Elife. 5:1-23. doi:10.7554/eLife.19071.

Pemberton, L.F., M.P. Rout, and G. Blobel. 1995. Disruption of the nucleoporin gene NUP133 results in clustering of nuclear pore complexes. Proc. Natl. Acad. Sci. U. S. A. 92:11871191. doi:10.1073/pnas.92.4.1187.

Raices, M., and M.A. D’Angelo. 2017. Nuclear pore complexes and regulation of gene expression. Curr. Opin. Cell Biol. 46:26-32. doi:https://doi.org/10.1016/j.ceb.2016.12.006.

Rhind, N., Z. Chen, M. Yassour, D.A. Thompson, B.J. Haas, N. Habib, I. Wapinski, S. Roy, M.F. Lin, D.I. Heiman, S.K. Young, K. Furuya, Y. Guo, A. Pidoux, H.M. Chen, B. Robbertse, J.M. Goldberg, K. Aoki, E.H. Bayne, A.M. Berlin, C.A. Desjardins, E. Dobbs, L. Dukaj, L. Fan, M.G. FitzGerald, C. French, S. Gujja, K. Hansen, D. Keifenheim, J.Z. Levin, R.A. Mosher, C.A. Müller, J. Pfiffner, M. Priest, C. Russ, A. Smialowska, P. Swoboda, S.M. Sykes, M. Vaughn, S. Vengrova, R. Yoder, Q. Zeng, R. Allshire, D. Baulcombe, B.W. Birren, W. Brown, K. Ekwall, M. Kellis, J. Leatherwood, H. Levin, H. Margalit, R. Martienssen, C.A. Nieduszynski, J.W. Spatafora, N. Friedman, J.Z. Dalgaard, P. Baumann, H. Niki, A. Regev, and C. Nusbaum. 2011. Comparative functional genomics of the fission yeasts. Science (80-. ). 332:930-936. doi:10.1126/science.1203357.

Schreiner, S.M., P.K. Koo, Y. Zhao, S.G.J. Mochrie, and M.C. King. 2015. The tethering of chromatin to the nuclear envelope supports nuclear mechanics. Nat. Commun. 6:1-13. 
doi:10.1038/ncomms8159.

Steglich, B., G.J. Filion, B. van Steensel, and K. Ekwall. 2012. The inner nuclear membrane proteins Man1 and Ima1 link to two different types of chromatin at the nuclear periphery in S. pombe. Nucleus. 3:77-87. doi:10.4161/nucl.18825.

Taddei, A., and S.M. Gasser. 2012. Structure and function in the budding yeast nucleus. Genetics. 192:107-129. doi:10.1534/genetics.112.140608.

Tan-Wong, S.M., H.D. Wijayatilake, and N.J. Proudfoot. 2009. Gene loops function to maintain transcriptional memory through interaction with the nuclear pore complex. Genes Dev. 23:2610-2624. doi:10.1101/gad.1823209.

Thaller, D.J., M. Allegretti, S. Borah, P. Ronchi, M. Beck, and C.P. Lusk. 2019. An escrt-lem protein surveillance system is poised to directly monitor the nuclear envelope and nuclear transport system. Elife. 8. doi:10.7554/eLife.45284.

Thaller, D.J., and C. Patrick Lusk. 2018. Fantastic nuclear envelope herniations and where to find them. Biochem. Soc. Trans. 46:877-889. doi:10.1042/BST20170442.

Ungricht, R., and U. Kutay. 2015. Establishment of NE asymmetry-targeting of membrane proteins to the inner nuclear membrane. Curr. Opin. Cell Biol. 34:135-141. doi:10.1016/j.ceb.2015.04.005.

Ungricht, R., and U. Kutay. 2017. Mechanisms and functions of nuclear envelope remodelling. Nat. Rev. Mol. Cell Biol. 18:229-245. doi:10.1038/nrm.2016.153.

Webster, B.M., P. Colombi, J. Jäger, and C. Patrick Lusk. 2014. Surveillance of nuclear pore complex assembly by ESCRT-IIINps4. Cell. 159:388-401. doi:10.1016/j.cell.2014.09.012.

Webster, B.M., D.J. Thaller, J. Jäger, S.E. Ochmann, S. Borah, and C.P. Lusk. 2016. Chm7 and Heh1 collaborate to link nuclear pore complex quality control with nuclear envelope sealing. EMBO J. 35. doi:10.15252/embj.201694574.

Xie, W., and B. Burke. 2017. Nuclear networking. Nucleus. 8:323-330. doi:10.1080/19491034.2017.1296616.

Yam, C., Y. Gu, and S. Oliferenko. 2013. Partitioning and remodeling of the Schizosaccharomyces japonicus mitotic nucleus require chromosome tethers. Curr. Biol. 23:2303-2310. doi:10.1016/j.cub.2013.09.057. 
bioRxiv preprint doi: https://doi.org/10.1101/2020.06.29.178129; this version posted June 30, 2020. The copyright holder for this preprint

(which was not certified by peer review) is the author/funder, who has granted bioRxiv a license to display the preprint in perpetuity. It is made available under aCC-BY-NC-ND 4.0 International license.

810 Yewdell, W.T., P. Colombi, T. Makhnevych, and C.P. Lusk. 2011. Lumenal interactions in

811 nuclear pore complex assembly and stability. Mol. Biol. Cell. 22:1375-1388.

812 doi:10.1091/mbc.E10-06-0554.

813 Zhang, Y., N.D. Serratore, and S.D. Briggs. 2017. N-ICE plasmids for generating N-terminal $3 \times$

814 FLAG tagged genes that allow inducible, constitutive or endogenous expression in

815 Saccharomyces cerevisiae. Yeast. 34:223-235. doi:10.1002/yea.3226. 
bioRxiv preprint doi: https://doi.org/10.1101/2020.06.29.178129; this version posted June 30, 2020. The copyright holder for this preprint (which was not certified by peer review) is the author/funder, who has granted bioRxiv a license to display the preprint in perpetuity. It is made

\section{Borah et al., Figure 1} available under aCC-BY-NC-ND 4.0 International license.
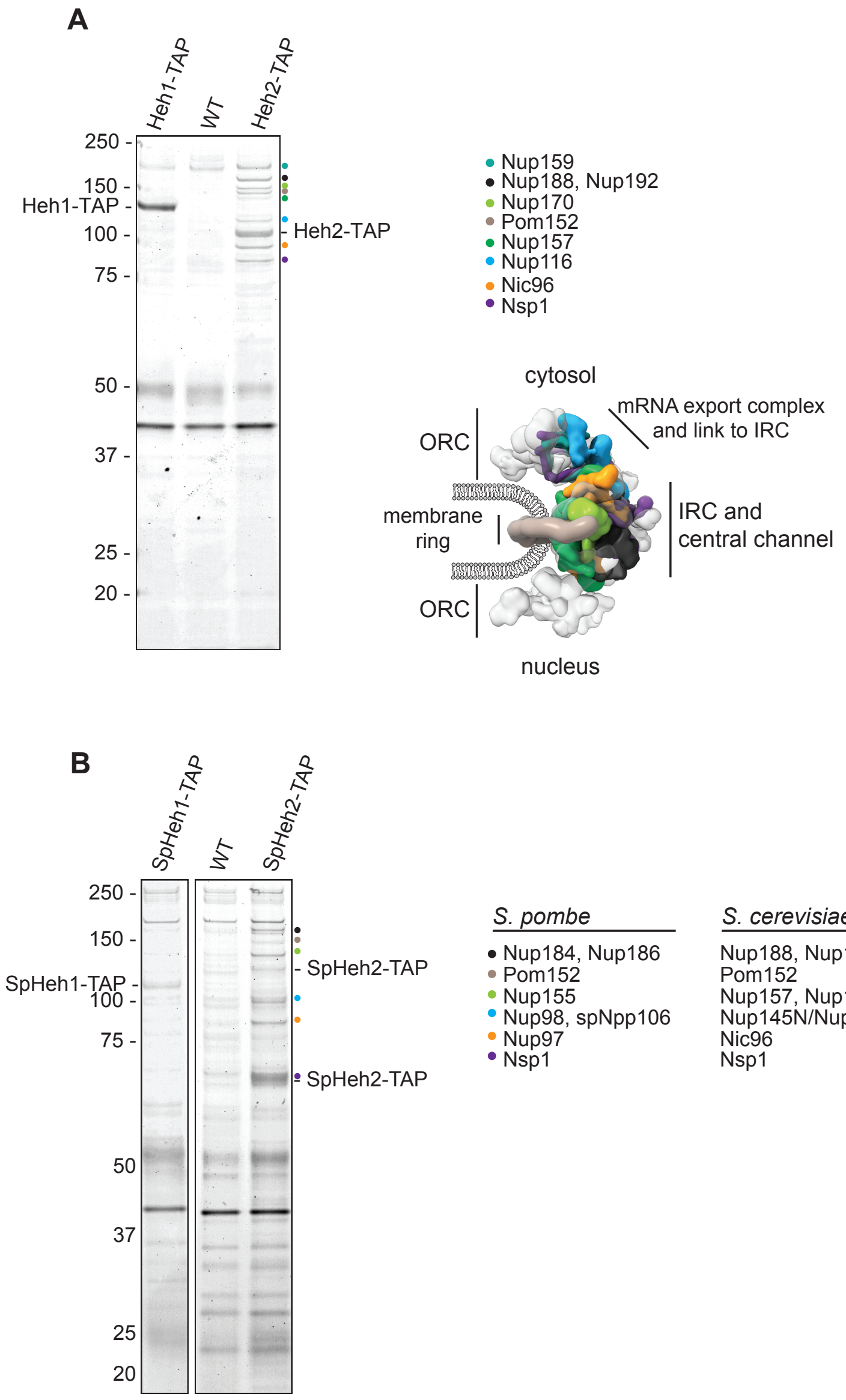

S. pombe

- Nup184, Nup186

- Pom152

- Nup155

- Nup98, spNpp106

- Nup97

- Nsp1
S. cerevisiae homologues

Nup188, Nup192

Pom152

Nup157, Nup170

Nup145N/Nup116/Nup100, Nic96

Nic96

Nsp1 
bioRxiv preprint doi: https://doi.org/10.1101/2020.06.29.178129; this version posted June 30, 2020. The copyright holder for this preprint (which was not certified by peer review) is the author/funder, who has granted bioRxiv a license to display the preprint in perpetuity. It is made available under aCC-BY-NC-ND 4.0 International license.

\section{Borah et al., Figure 2}

A
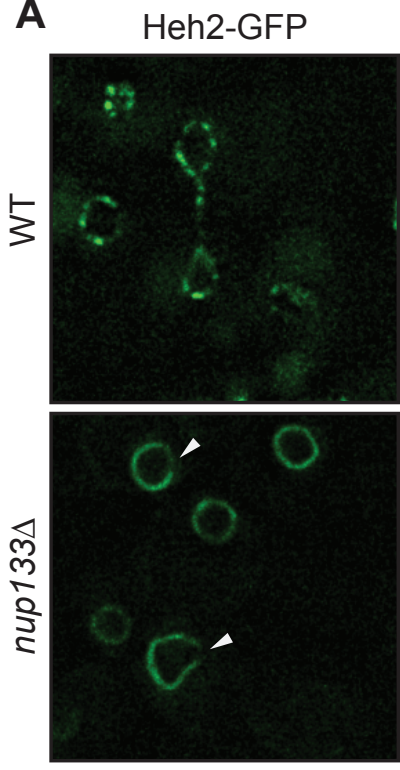

C
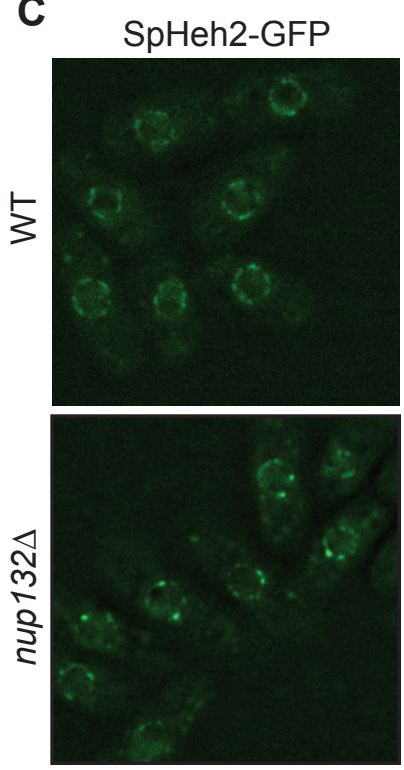

Nup82-mCherry
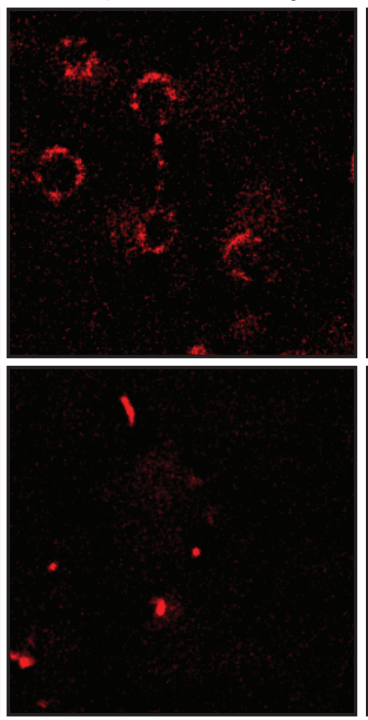

SpNup107-mCherry
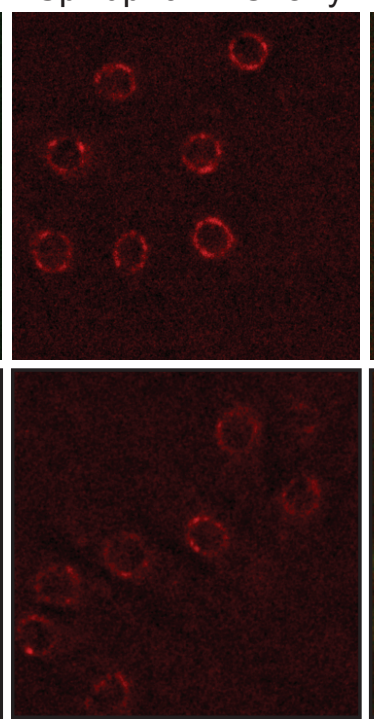

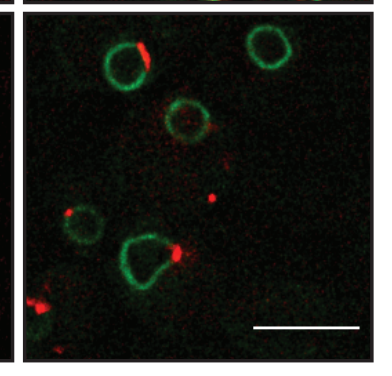

Merge

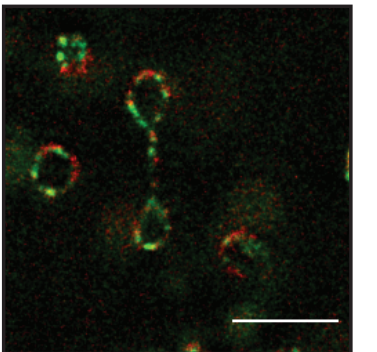

Merge

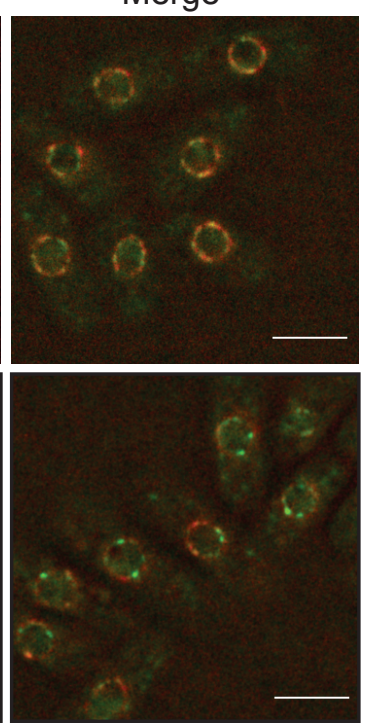

B
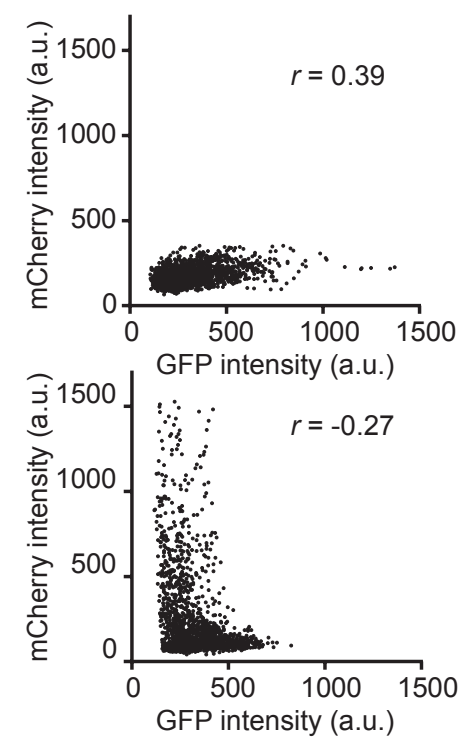

D
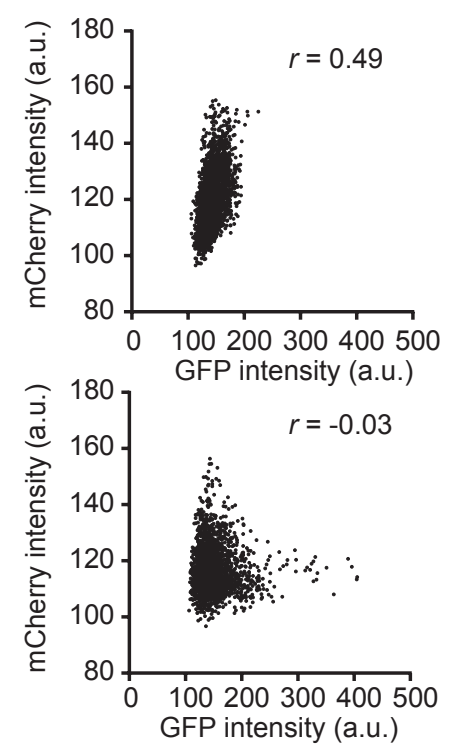


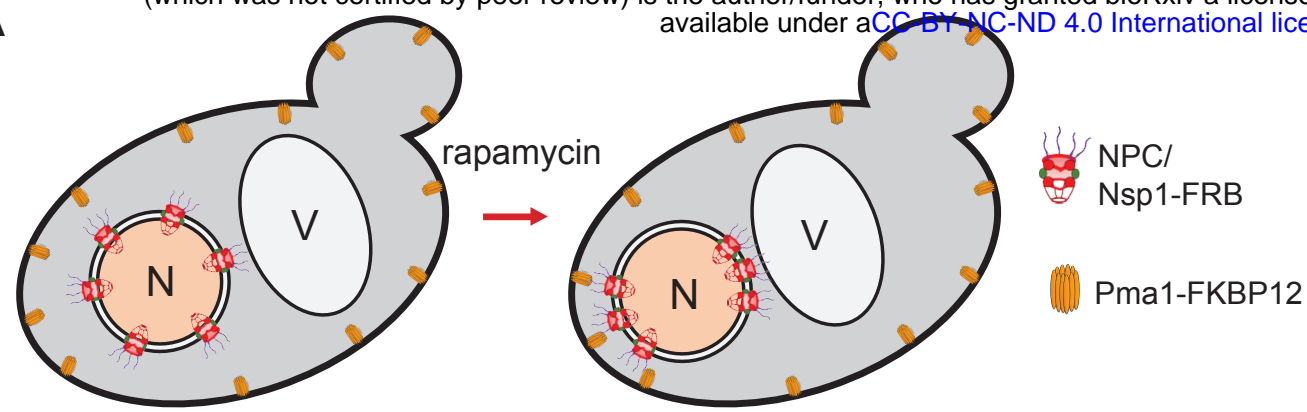

B

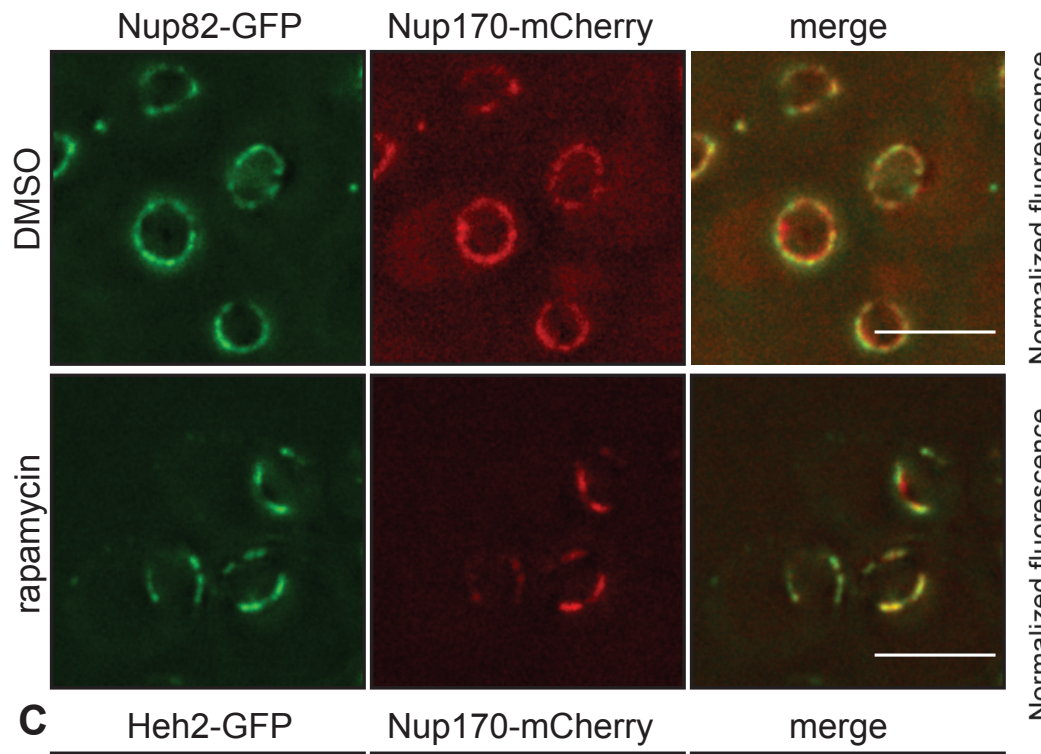

C
O

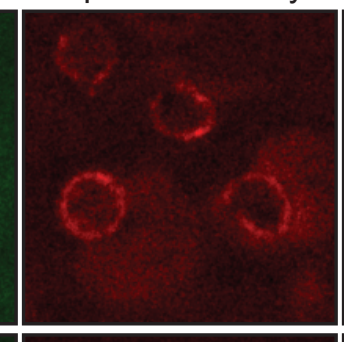

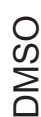

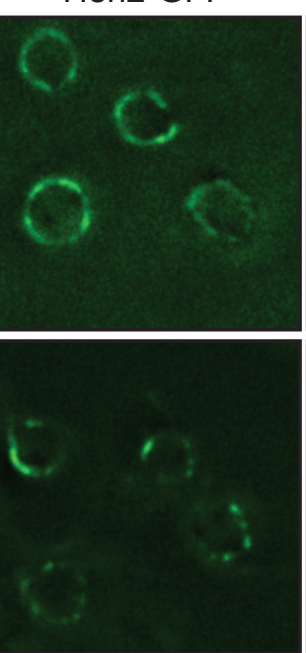

D

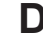

Heh1-GFP

Nup170-mCherry
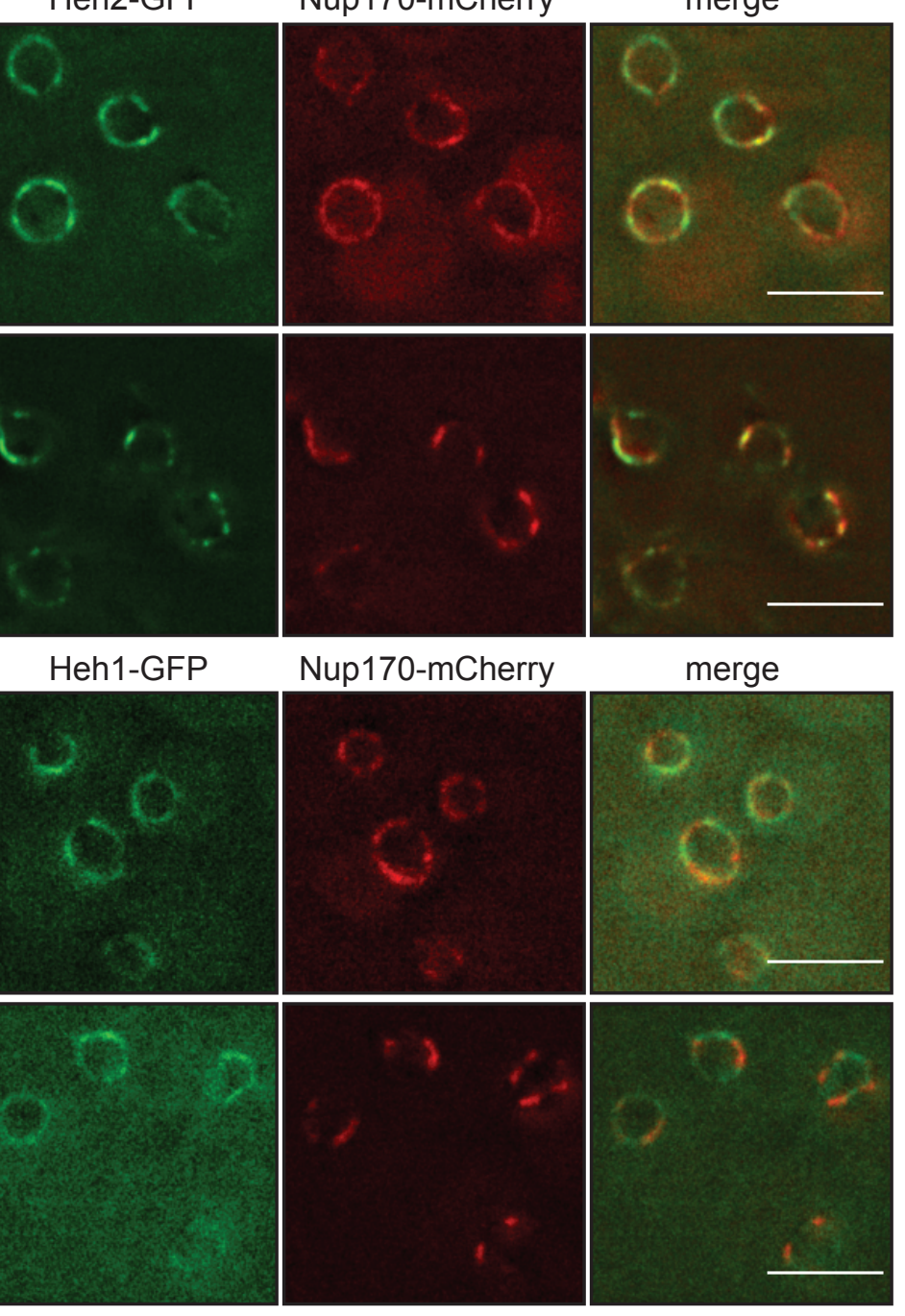

Nsp1-FRB Pma1-FKBP12
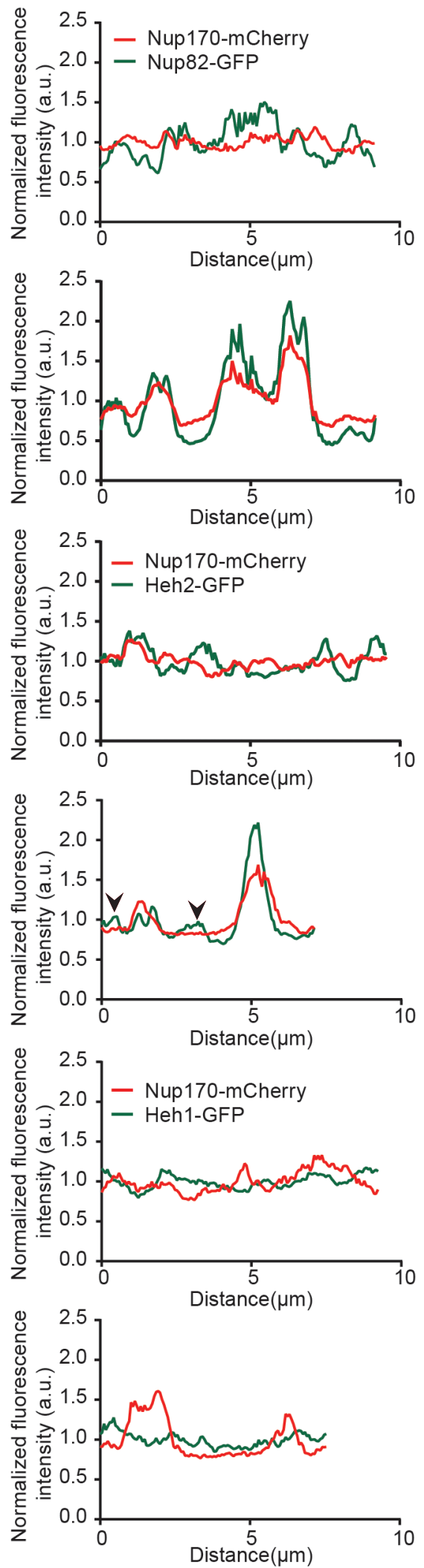
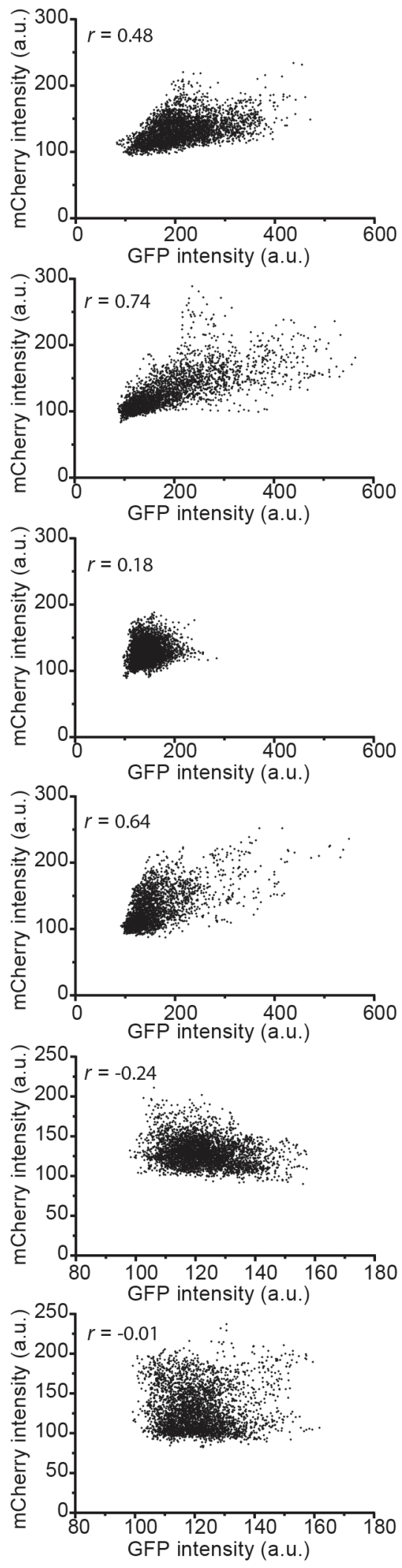
RorbinRxił preprintelpi:https://gpi.org/10.1101/2020.06.29.178129; this version posted June 30, 2020. The copyright holder for this preprint

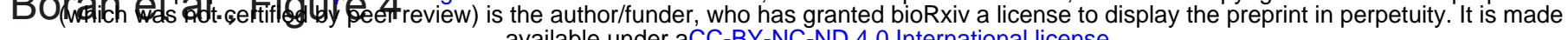

A available under aCC-BY-NC-ND 4.0 International license.

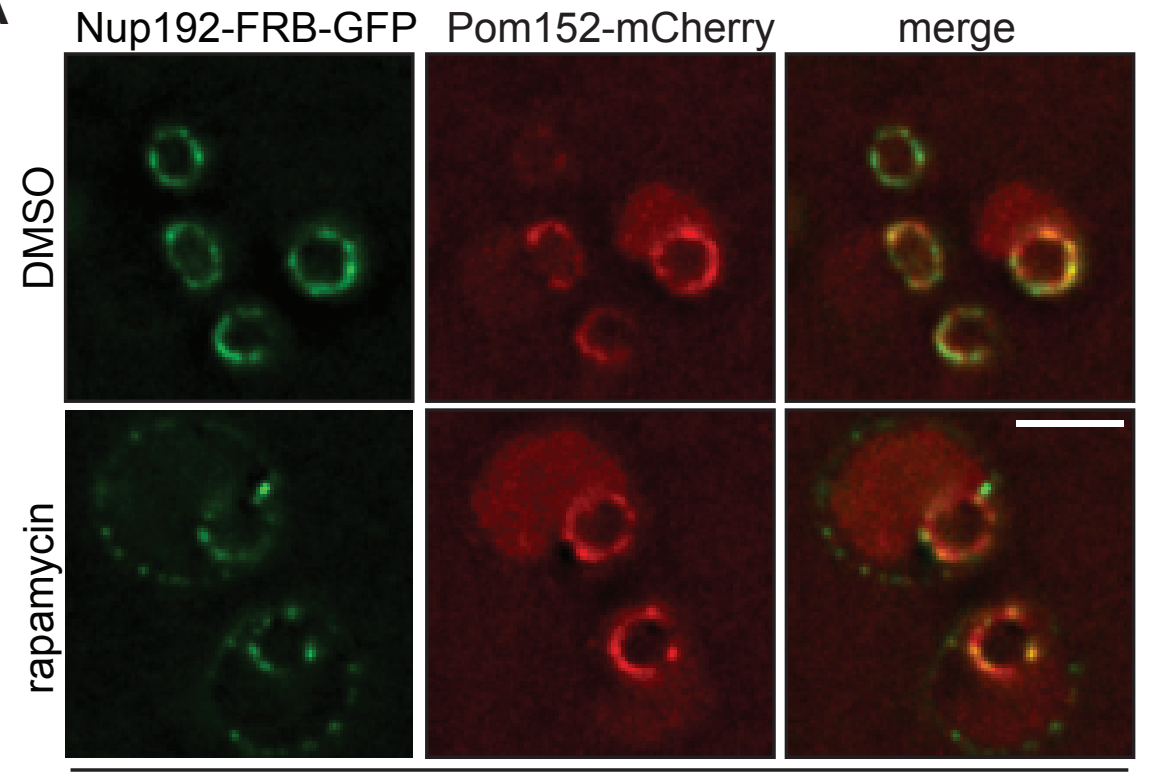

Pma1-FKBP12

B

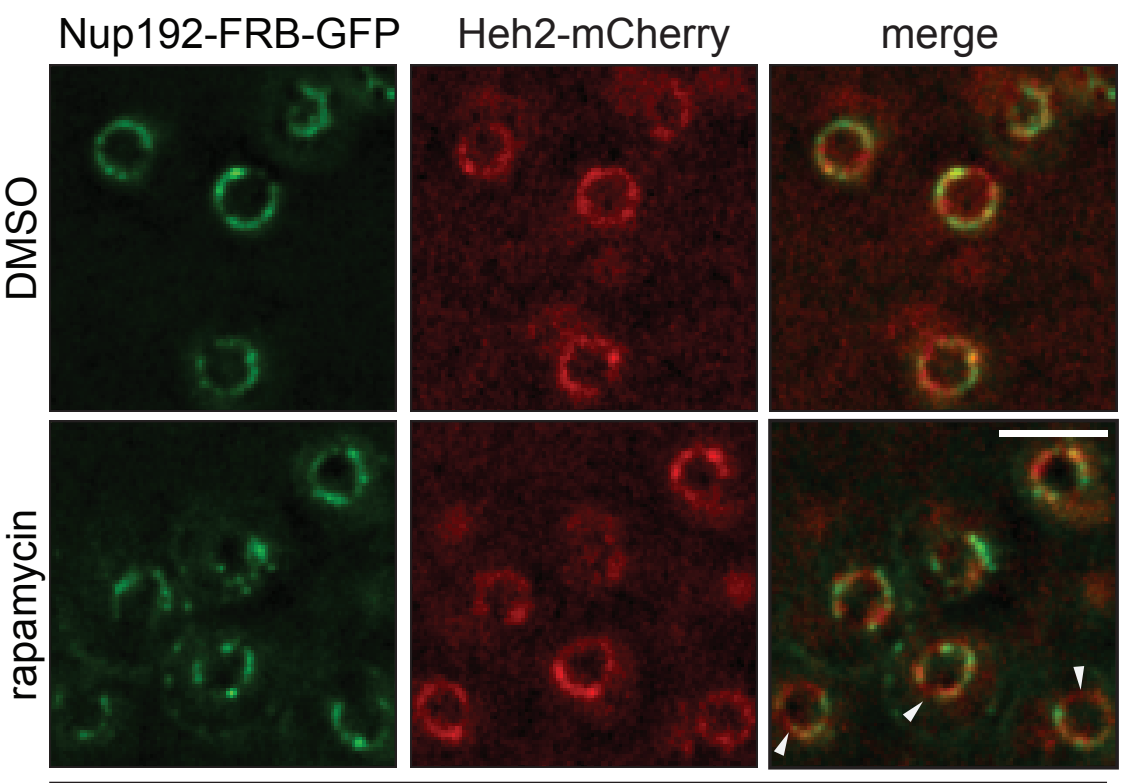

Pma1-FKBP12

C

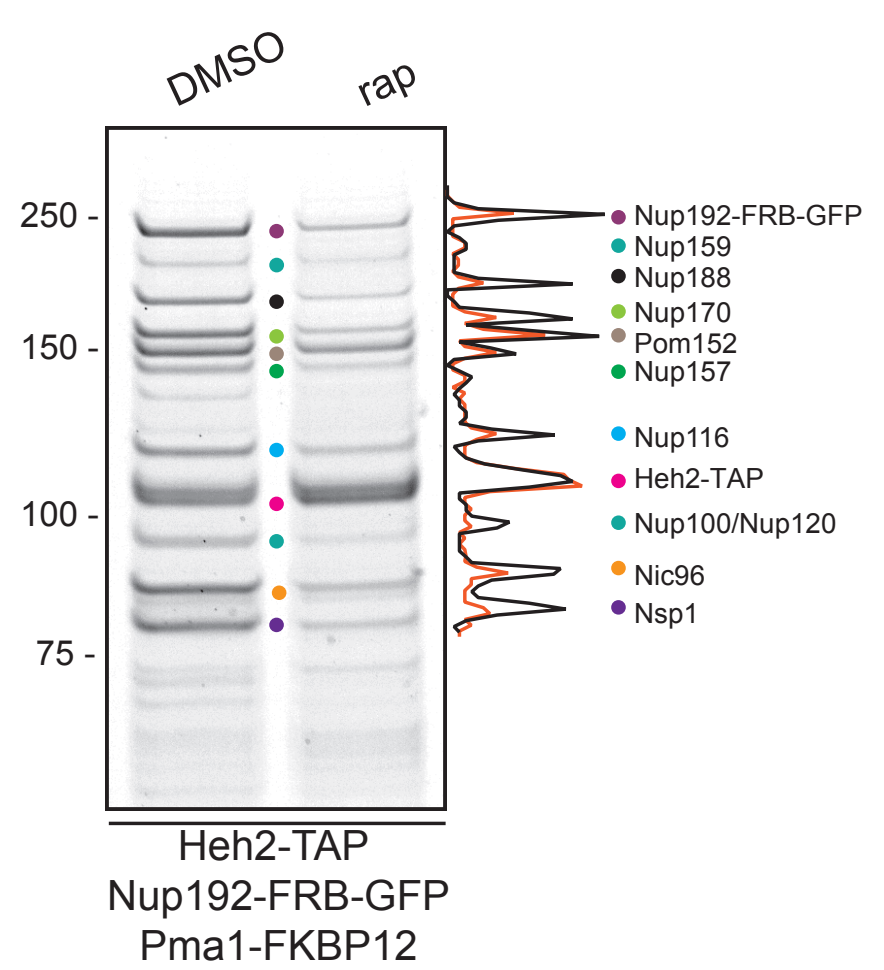

Heh2-TAP

Nup192-FRB-GFP

Pma1-FKBP12

C
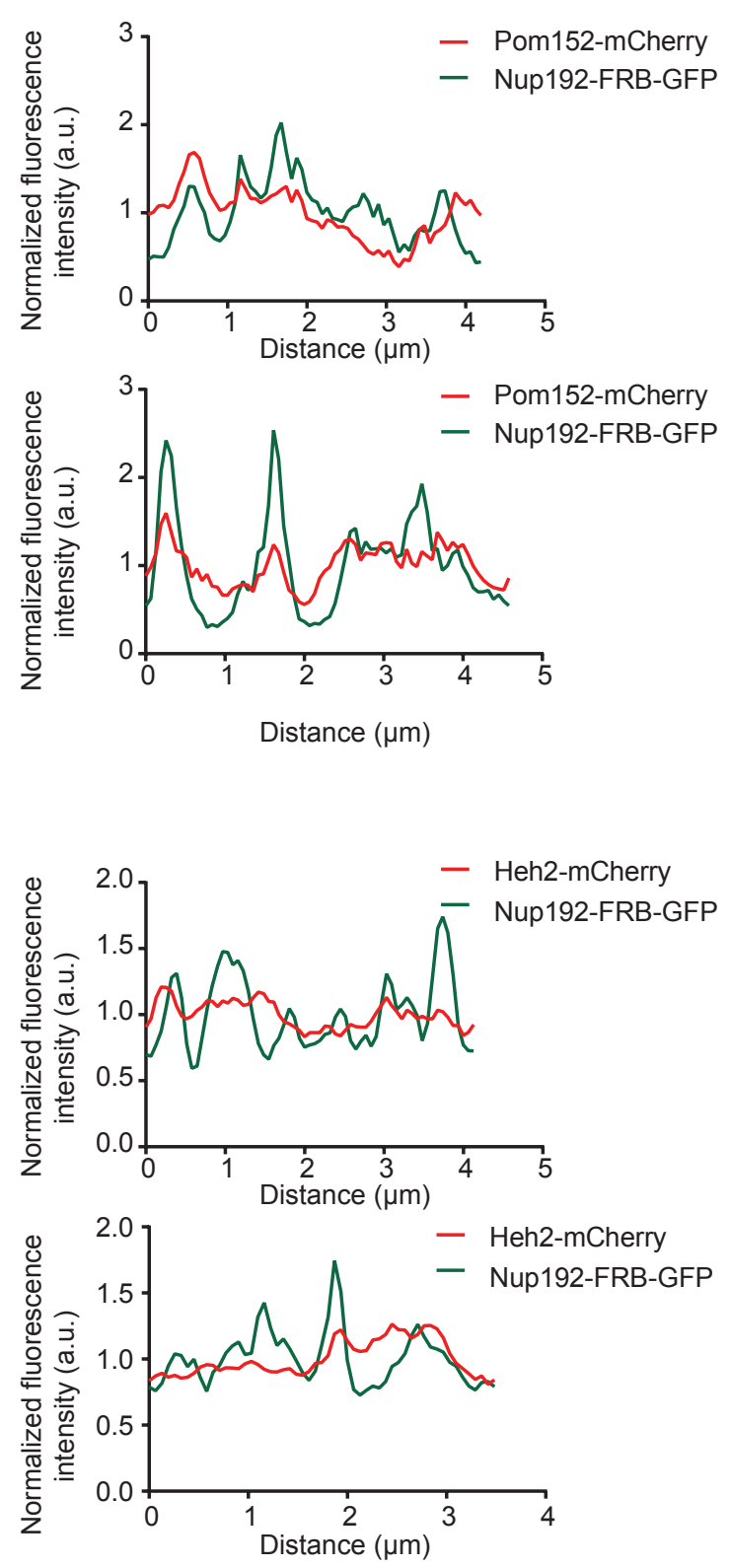
bioRxiv preprint doi: https://doi.org/10.1101/2020.06.29.178129; this version posted June 30, 2020. The copyright holder for this preprint

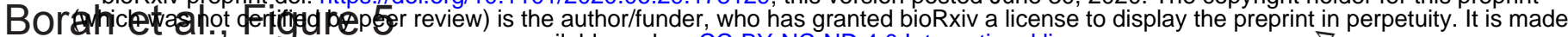

A

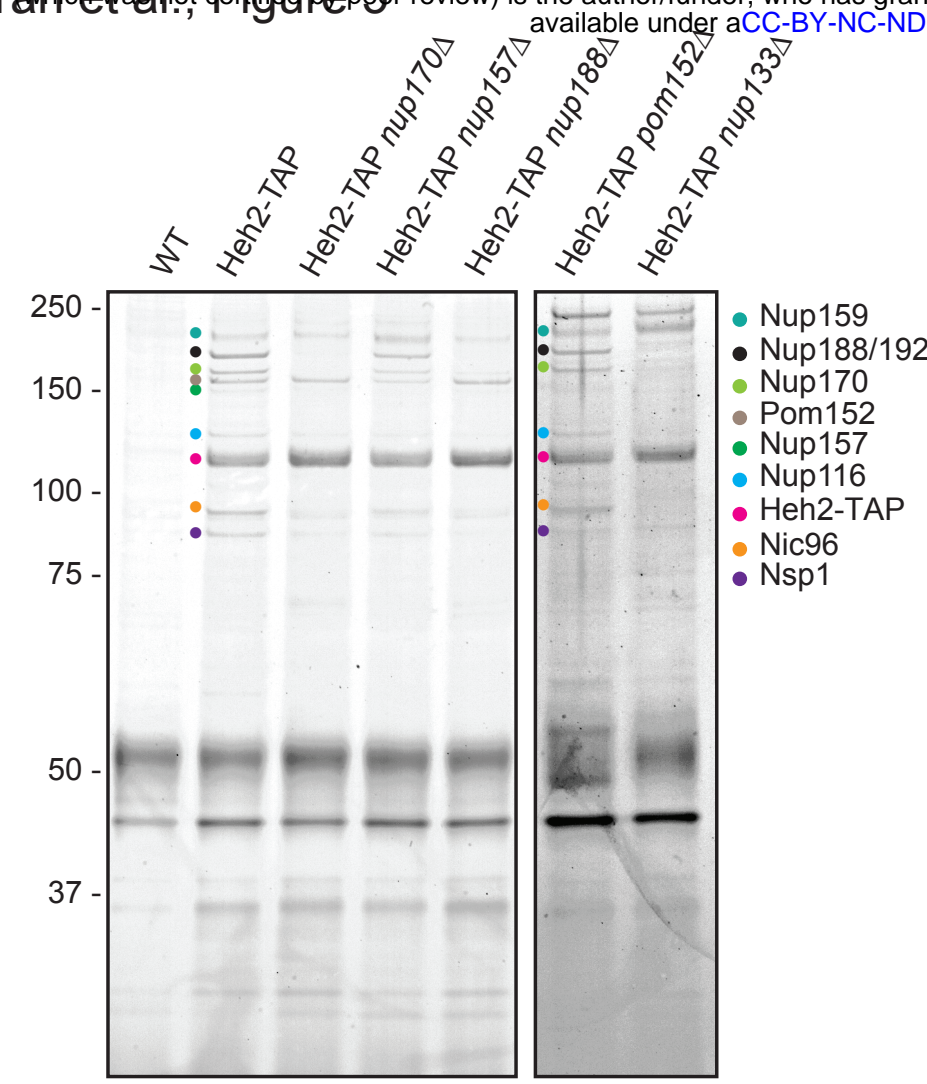

B
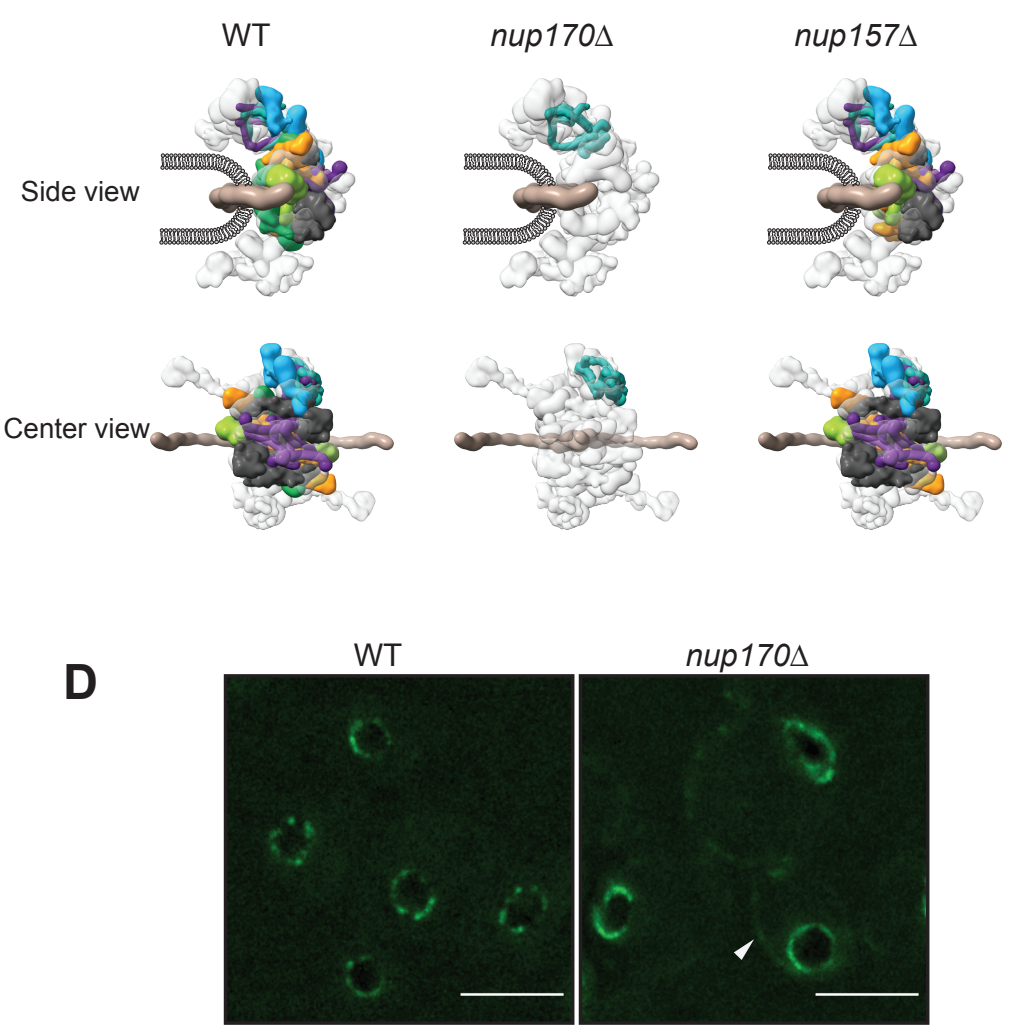

nup188А

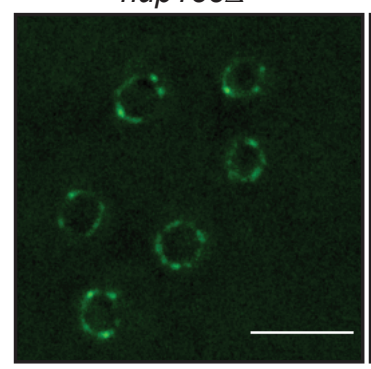

pom152ム

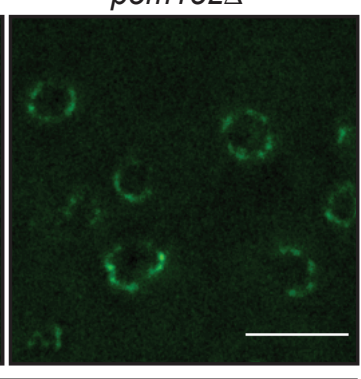

Heh2-GFP
C

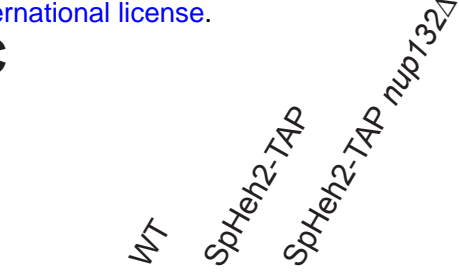
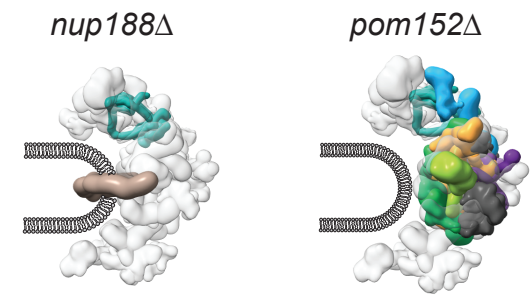

nup $133 \Delta$
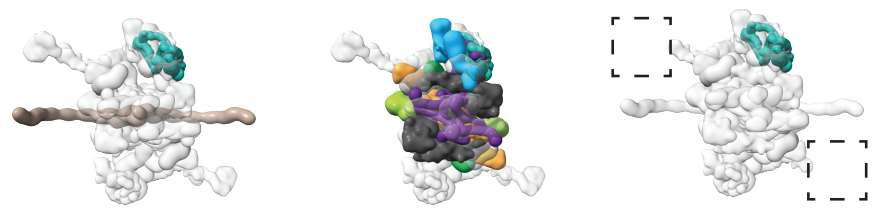

- Nup98, Npp106

- Nup97

- SpHeh2-TAP, Nsp1

37 -

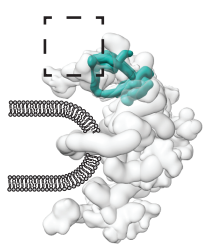


bioRxiv preprint doi: https://doi.org/10.1101/2020.06.29.178129; this version posted June 30, 2020. The copyright holder for this preprint (which was not certified by peer review) is the author/funder, who has granted bioRxiv a license to display the preprint in perpetuity. It is made Borah et al., Figure 6

A

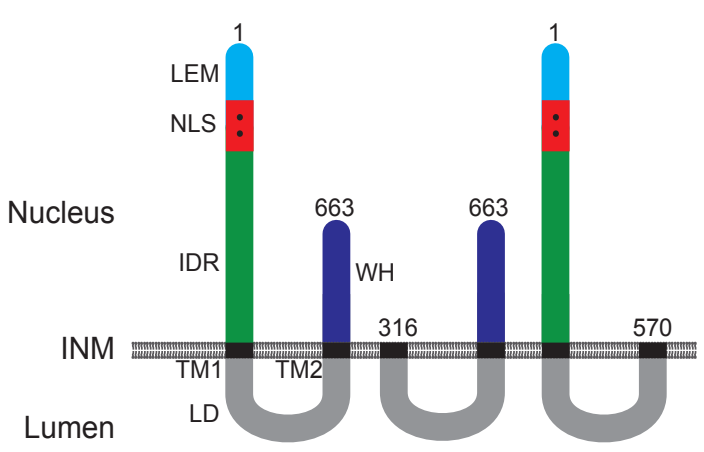

C

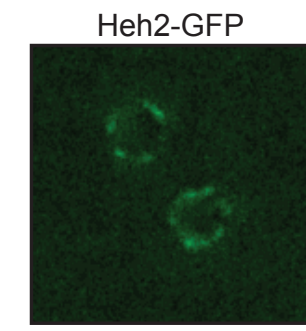

heh2-(1-570)-GFP

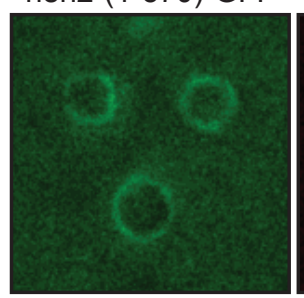

E

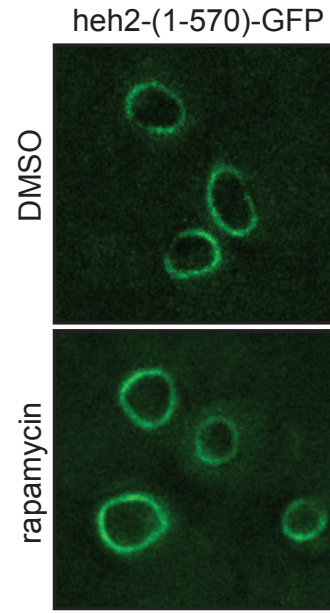

G

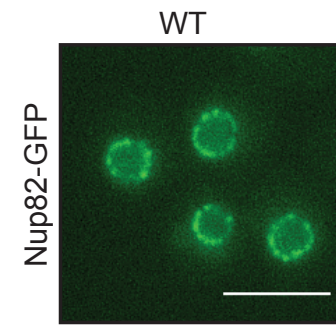

I

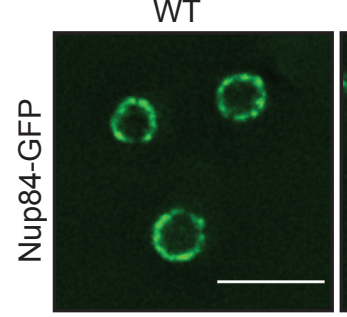

Nup82-mCherry

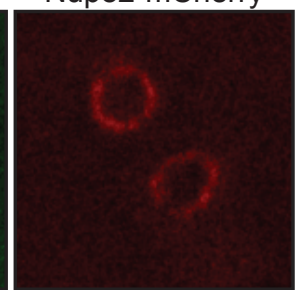

Nup82-mCherry

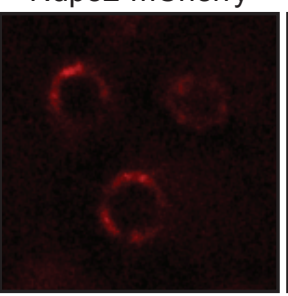

Nup170-mCherry

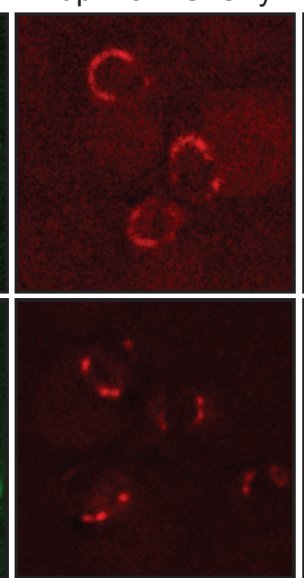

heh2 $\Delta$

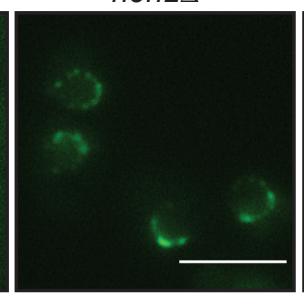

heh2-(316-663)

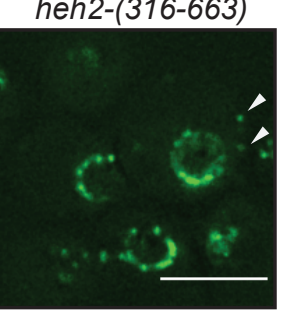

merge

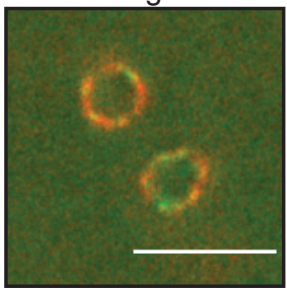

merge

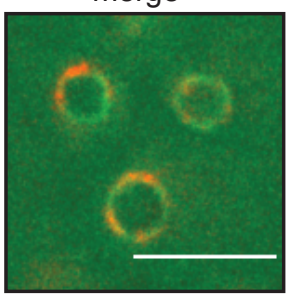

merge

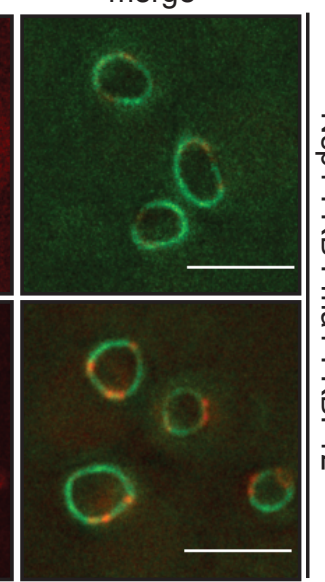

heh2-(1-570)

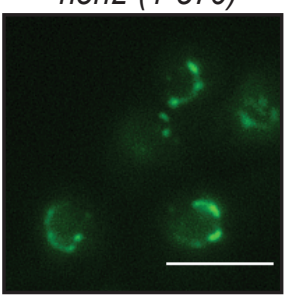

J

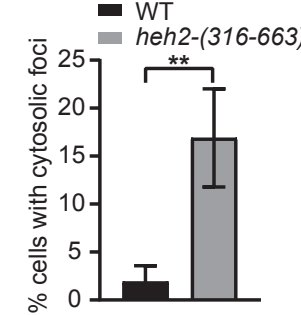

B

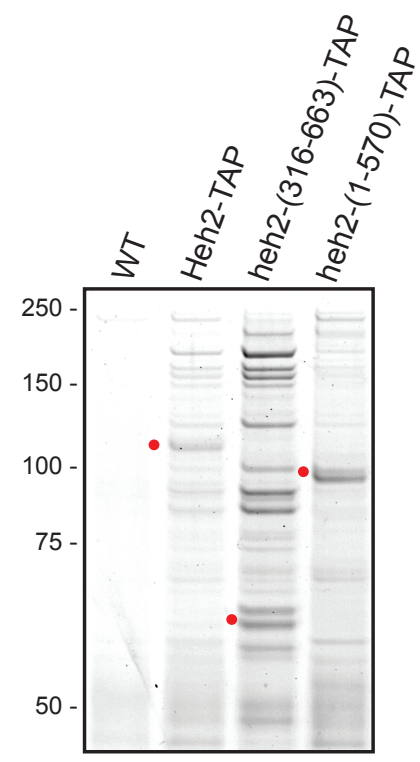

D

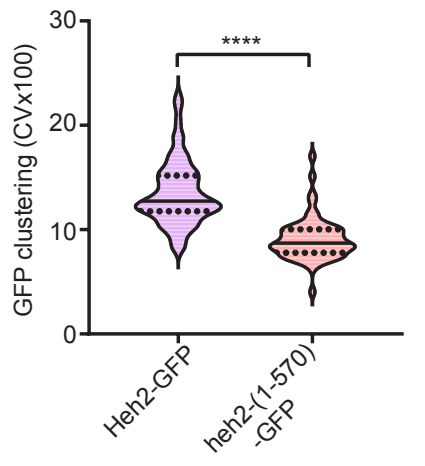

F
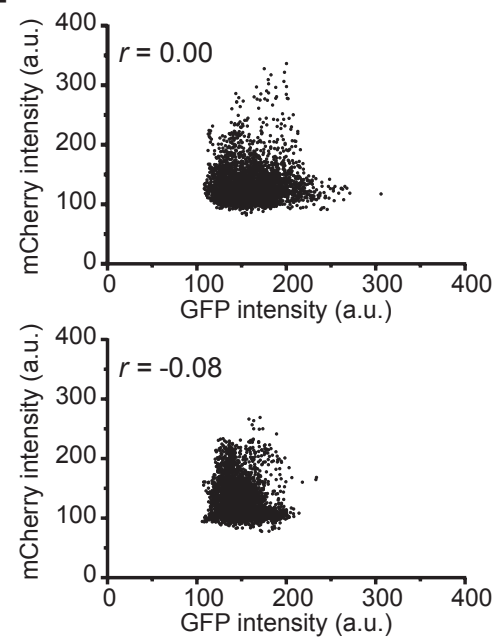

H

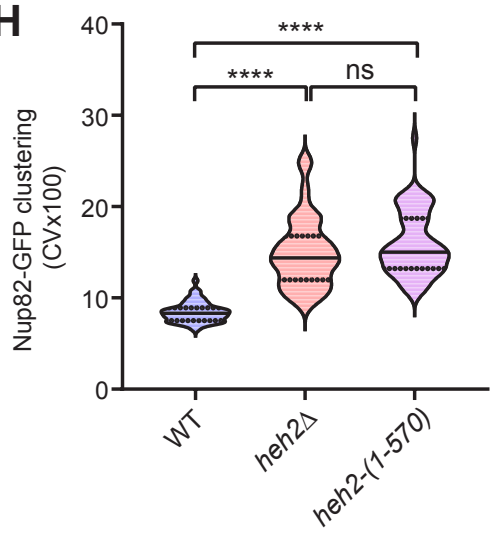


Table S1. Yeast strains

ins

\begin{tabular}{|c|c|c|c|}
\hline Name & Genotype & Origin & Generation \\
\hline W303a & MATa, ade2-1 can1-100 HIS33-11,15 leu2-3,112 trp1-1 ura3-1 & EUROSCARF & \\
\hline W303a & MATa, ade2-1 can1-100 HIS33-11,15 leu2-3,112 trp1-1 ura3-1 & EUROSCARF & \\
\hline CPL111 & W303, heh24::kanMX6 & This study & \\
\hline CPL112 & W303, heh24::kanMX6 & This study & \\
\hline SBCPL42 & Heh2-TAP::HIS & Dharmacon & \\
\hline SBCPL54 & W303, HEH2-TAP::TRP1 & This study & Integration through PCR product transformation \\
\hline SBCPL174 & W303, HEH1-TAP::HIS33 & Dharmacon & \\
\hline SBCPL64 & W303, heh2 (1-571)-TAP::KAN & This study & Integration through PCR product transformation \\
\hline SBCPL122 & W303, 3xFLAG heh2(316-663)-TAP:::TRP & This study & N-terminal 3×FLAG integration through PCR product transformation, Zhang et al., 2017 \\
\hline SBCPL76 & W303, heh2А::kanMX6 Nup82-GFP::TRP & This study & Integration through PCR product transformation \\
\hline SBCPL75 & W303, heh2(1-571)-TAP::KAN NUP82-GFP:: TRP & This study & Integration through PCR product transformation in SBCPL64 \\
\hline SBCPL88 & W303,HEH2-3HA-GFP::hphMX6 & This study & Integration through PCR product transformation \\
\hline SBCPL89 & W303,HEH2-3HA-GFP::hphMX6 & This study & Integration through PCR product transformation \\
\hline SBCPL139 & W303, HEH2-3HA-GFP:hphMX6 NUP82-mCherry:natMX6 & This study & Progeny from cross between SBCPL138 and CVCPL109 \\
\hline SBCPL96 & W303, HEH2-TAP:: TRP nup $170 \Delta::$ natMX6 & This study & Progeny from cross between SBCPL54 and CPL634 \\
\hline SBCPL56 & W303, HEH2-TAP::TRP nup $188 \Delta:: K A N$ & This study & Progeny from cross between SBCPL54 and CPL766 \\
\hline SBCPL169 & W303, HEH2-TAP::TRP nUp $157 \triangle::$ hphMX 6 & This study & Progeny from cross between SBCPL54 and PCCPL240 \\
\hline SBCPL61 & W303, HEH2-TAP::hphMX6 nup 133 $1:$ kan & This study & Progeny from cross between SBCPL54 and CPL337 \\
\hline SBCPL170 & W303, HEH2-TAP-TAP::TAP pom152 $\triangle:$ kan & This study & Progeny from cross between SBCPL55 and CPL398 \\
\hline SBCPL138 & W303, HEH2-3HA-GFP::hphMX6 nup133د::KAN & This study & \\
\hline SBCPL145 & 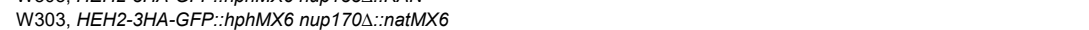 & This study & Progeny from cross between SBCPL89 and CPL634 \\
\hline SBCPL150 & W303, HEH2-3HA-GFP::hphMX6 pom152ム::KAN & This study & Progeny from cross between SBCPL88 and CPL399 \\
\hline SBCPL157 & W303, HEH2-3HA-GFP::hPhMX6 nup188А::KAN & This study & \\
\hline SBCPL140 & W303, HEH2-3HA-GFP::hphMX6 NUP82-mCherry::natMX6 nup133А::KAN & This study & Progeny from cross between SBCPL138 and CVCPL109 \\
\hline HHY110 & W303, MAT alpha tor1-1 fpr1::natMX6MX6 PMA1-2×FKBP12::TRP1. & $\begin{array}{l}\text { Inis stuay (Haruki et al., 2008) } \\
\text { Euroscarf (hat }\end{array}$ & \\
\hline CPL1230 & HHY110, NSP1-FRB-GFP::HIS33MX6 NUP170-mCherry:kanMX6 & This study & PCR-based integration using pFA6a-mCherry-kanMX6 and pFA6a-FRB-GFP-His3MX6 \\
\hline SBCPI 84 & 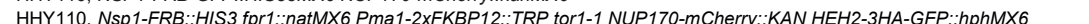 & This studv & 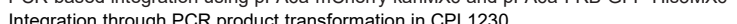 \\
\hline SBCPL85 & HHY110, Nsp1-FRB:::HIS3 fpr1::natMX6 Pma1-2XFKBP12:: TRP tor1-1 NUP170-mCherry::KAN HEH1-3HA-GFP:: hphMX6 & This study & $\begin{array}{l}\text { Integration through PCR product transformation in CPL } 1230 \\
\text { Int }\end{array}$ \\
\hline SBCPL86 & HHY110, NSP1-FRB:: HIS3 fpr1:: natMX6 Pma1-2xFKBP12:: TRP tor1-1 NUP170-mCherry::KAN NUP82-3HA-GFP::"hphMX6 & This study & Integration through PCR product transformation in CPL 1230 \\
\hline SBCPL109 & HHY110, NSP1-FRB::HIS3 fpr1::natMX6 Pma1-2xFKBP12::TRP tor1-1 NUP170-mCherry::KAN heh2(1-570)-3HA-GFP::hphMX6 & This study & \\
\hline SBCPL63 & HHY110, HEH2-TAP::KAN Nup192-3xHA-FRB-GFP::HIS3 Pma1-2xFKBP12::TRP fpr1::nat & This study & Integration through PCR product transformation in DTCPL1539 \\
\hline DTCPL1846 & HHY110, NUP192-3XHA-FRB-GFP::his3 POM152-mCherry::kanMX6 PMA1-2xFK & This study & Progeny from cross between DTCPL1539 and DTCPL1645 \\
\hline \multirow{2}{*}{\multicolumn{4}{|c|}{$\begin{array}{l}\text { DTCPL 1881 HHY110, NUP192-3XHA-FRB-GFP::'his3 HEH2-3xHA-mCherry::kanMM6 PMA1-2XFKBP12::Th } \\
\text { Schizosaccharomyces pombe strains }\end{array}$}} \\
\hline & & & \\
\hline MKSP399 & $h+$ leu1-32 ura4-D18 & This study & \\
\hline MKSP3045 & $h+$ Heh2-TAP::HygR leu1-32 ura4-D18 & This study & Integration through PCR product transformation \\
\hline MKSP3049 & $h$ ? Heh2-TAP:::HygR nup 132::KanR leu1-32 ura4-D18 & This study & Progeny from cross between MKSP3045 and MKSP264 \\
\hline MKSP3071 & h? Heh2-GFP:HygR Nup107-mCherry::NatR leu1-32 ura4-D18 & This study & Progeny from cross between MKSP 1410 and MKSP 1118 \\
\hline MKSP3090 & $h+$ Heh2-GFP:HygR Nup107-mCherry::NatR nup132::KanR leu1-32 ura4-D18 & This study & Progeny from cross between MKSP3071 and MKSP264 \\
\hline
\end{tabular}

$\begin{array}{lll}\text { MKSP3090 } & \text { h+ Heh2-GFP:HygR Nup107-mCherry::NatR nup132::KanR leu1-32 ura4-D18 } & \text { This study } \\ & & \text { This study }\end{array}$

Progeny from cross 
bioRxiv preprint doi: https://doi.org/10.1101/2020.06.29.178129; this version posted June 30,2020 . The copyright holder for this preprint (which was not certified by peer review) is the author/funder, who has granted bioRxiv a license to display the preprint in perpetuity. It is made available under aCC-BY-NC-ND 4.0 International license.

Table S2. Plasmids

\begin{tabular}{lll}
\hline Name & Description & Source \\
\hline pFA6a-GFP-his3MX6 & Template for PCR based chromosomal integration of GFP ORF & Longtine et al., 1998 \\
pFA6a-GFP-natMX6 & Template for PCR based chromosomal integration of GFP ORF & Van Driessche et al., 2005 \\
pFA6a-GFP-kanMX6 & Template for PCR based chromosomal integration of GFP ORF & Longtine et al., 1998 \\
pFA6a-hphMX6 & Template for PCR based chromosomal integration of hphMX6 cassette & Longtine et al., 1998 \\
pFA6a-natMX6 & Template for PCR based chromosomal integration of natMX6 cassette & Longtine et al., 1998 \\
pFA6a-kanMX6 & Template for PCR based chromosomal integration of kanMX6 cassette & Longtine et al., 1998 \\
pFA6a-mCherry-kanMX6 & Template for PCR based chromosomal integration of mCherry ORF & EUROSCARF \\
pFA6a-mCherry-natMX6 & Template for PCR based chromosomal integration of mCherry ORF & EUROSCARF \\
pSBCPL3 & pFA6a-TAP-his3MX6, template for PCR based chromosomal integration of TAP-TAG & This study \\
pSBCPL4 & pFA6a-TAP-TRP, template for PCR based chromosomal integration of TAP-TAG & This study \\
pK3F & N-ICE plasmid pK3F, for N-terminal 3×FLAG integration & Addgene \\
pSH47 & Cre recombinase under the GAL1 promoter & EUROSCARF \\
\hline
\end{tabular}

